\title{
Serpentes do estado de Rondônia, Brasil
}

\author{
Paulo Sérgio Bernarde ${ }^{1,4}$, Saymon de Albuquerque ${ }^{2}$, \\ Thiago Oliveira Barros ${ }^{3} \&$ Luiz Carlos Batista Turci ${ }^{1}$
}

\author{
${ }^{1}$ Laboratório de Herpetologia, Centro Multidisciplinar, Universidade Federal do Acre - UFAC, \\ Campus Floresta, CEP 69980-000, Cruzeiro do Sul, AC, Brasil \\ ${ }^{2}$ Departamento de Zoologia, Centro de Ensino, Faculdade São Lucas, Rua Alexandre Guimarães, 1927, \\ Bairro Areal, CEP 76804-373, Porto Velho, RO, Brasil \\ ${ }^{3}$ Faunística Soluções Ambientais - FSA, Brasília, DF, Brasil \\ ${ }^{4}$ Autor para correspondência: Paulo Sérgio Bernarde, e-mail: snakebernarde@hotmail.com
}

BERNARDE, P.S., ALBUQUERQUE, S., BARROS, T.O. \& TURCI, L.C.B. Snakes of Rondônia State, Brazil. Biota Neotrop. 12(3): http://www.biotaneotropica.org.br/v12n3/en/abstract?inventory+bn00412032012

\begin{abstract}
The present study aimed to update the snake species list of the state of Rondonia based on a literature review, and to provide information on species distribution in the state. A total of 118 snake species from eight families have been recorded in Rondônia: Leptotyphlopidae (4 species), Typhlopidae (1), Aniliidae (1), Boidae (6), Colubridae (21), Dipsadidae (67), Elapidae (9) and Viperidae (9). Of these, 109 species were recorded in areas of amazon forest and 27 in cerrado areas. The observed richness in cerrado areas is probably underestimated because of the low number of studies, and the small coverage (ca. 5\%) of this vegetation in the state. Nine species (Epicrates crassus, Chironius flavolineatus, Drymoluber brazili, Apostolepis striata, Oxyrhopus rhombifer, Pseudoboa nigra, Xenodon merremii, Bothrops mattogrossensis and Crotalus durissus) were recorded exclusively in cerrado areas, being related with this environment in Amazon. Six species (Masticophis mentovarius, Apostolepis striata, Erythrolamprus mimus, Micrurus mipartitus, Micrurus sp. and Bothrocophias microphthalmus) have been recorded in Brazil only in Rondônia. There are gaps of knowledge on snake fauna in some regions of the state, and further studies are essential. This is an emergencial measure, because of the increaseing habitat destruction along the Cerrado and southern portions of Amazonia.
\end{abstract}

Keywords: reptiles, squamata, amazon, inventory.

BERNARDE, P.S., ALBUQUERQUE, S., BARROS, T.O. \& TURCI, L.C.B. Serpentes do estado de Rondônia, Brasil. Biota Neotrop. 12(3): http://www.biotaneotropica.org.br/v12n3/pt/abstract?inventory+bn00412032012

Resumo: Esse estudo teve como objetivo atualizar a lista de serpentes do estado de Rondônia a partir de levantamento bibliográfico, e fornecer algumas informações sobre a distribuição das espécies. São registradas para o estado de Rondônia 118 espécies de serpentes, pertencentes a oito famílias: Leptotyphlopidae (4 espécies), Typhlopidae (1), Aniliidae (1), Boidae (6), Colubridae (21), Dipsadidae (67), Elapidae (9) e Viperidae (9). Dessas, 109 foram registradas para áreas de floresta amazônica e 27 em cerrado. A menor riqueza encontrada em cerrado (27 espécies) provavelmente deve estar associada aos poucos trabalhos desenvolvidos nessas áreas e pelo fato dessa formação vegetal ocupar uma área de cerca de apenas 5\% do estado. Nove espécies (Epicrates crassus, Chironius flavolineatus, Drymoluber brazili, Apostolepis striata, Oxyrhopus rhombifer, Pseudoboa nigra, Xenodon merremii, Bothrops mattogrossensis e Crotalus durissus) foram registradas exclusivamente em áreas de cerrado, sendo formas associadas a esse ambiente na Amazônia. Seis espécies (Masticophis mentovarius, Apostolepis striata, Erythrolamprus mimus, Micrurus mipartitus, Micrurus sp. e Bothrocophias microphthalmus) são conhecidas no Brasil apenas para Rondônia. Existem lacunas sobre o conhecimento das serpentes em algumas regiões de Rondônia, sendo essencial a realização de mais estudos de inventário. Tal necessidade se torna mais urgente devido à crescente destruição dos habitats ao longo do Cerrado e nas porções sul da Amazônia.

Palavras-chave: répteis, squamata, amazônia, inventário. 


\section{Introdução}

O Brasil apresenta 375 espécies de serpentes conhecidas (Bérnils \& Costa 2011), cerca de 150 delas ocorrendo na Amazônia, domínio com maior riqueza desse grupo (Rodrigues 2005, Avila-Pires et al. 2007). Os valores de riqueza, no entanto, são ainda subestimados, uma vez que muitas regiões permanecem subamostradas (Avila-Pires et al. 2007). À medida que aumentam os estudos, novos registros são feitos para o Brasil (e. g., Bernarde et al. 2011a) e novas espécies são descritas (e. g., Prudente \& Passos 2008, 2010, Zaher et al. 2008). Na Amazônia brasileira, estudos sobre comunidades e levantamento de espécies de serpentes foram desenvolvidos principalmente no estado do Amazonas (e. g., Zimmermann \& Rodrigues 1990, Martins \& Oliveira 1998, Vogt et al. 2007, Avila-Pires et al. 2009, Prudente et al. 2010), Pará (Nascimento et al. 1987, Cunha \& Nascimento 1978, 1993, Frota 2004, Frota et al. 2005, Prudente \& Santos-Costa 2005, Avila-Pires et al. 2009, 2010) e Rondônia (e. g., Amaral 1925, Vanzolini 1986, Nascimento et al. 1988, Jorge-da-Silva 1993, Bernarde \& Abe 2006, Avila-Pires et al. 2009). Entretanto, mesmo nesses estados existem ainda amplas regiões pouco estudadas.

A primeira contribuição sobre as serpentes de Rondônia corresponde aos espécimes coletados entre 1908 e 1914 pela "Comissão de Linhas Telegráficas Estratégias do Mato Grosso ao Amazonas" coordenada pelo Marechal Rondon, cujo material foi analisado por Afrânio do Amaral (Amaral 1925, 1948). Nessas publicações, não são fornecidas as localidades da maioria dos espécimes, mas grande parte do material foi coletado em área do que corresponde atualmente ao estado de Rondônia (Jorge-da-Silva 1993). Amaral (1925) apresentou uma lista contendo algumas espécies raras (e. g., Rhinobotryum lentiginosum) e descreveu três novas espécies (Apostolepis rondoni, Liophis longiventris e Micrurus albicinctus), sendo que $A$. rondoni é considerada sinônima de $A$. nigrolineata (Lema \& Albuquerque 2010).

Na Década de 1980 foi criado o "Programa de Desenvolvimento Integrado do Noroeste do Brasil (Polonoroeste). Devido ao ritmo acelerado da colonização com seus impactos ambientais, o Conselho Nacional de Desenvolvimento Científico e Tecnológico (CNPq) dentro desse programa ficou responsável pelo diagnóstico dos recursos naturais da região (Vanzolini 1986). Durante o Programa Polonoroeste, dois inventários herpetofaunísticos conduzidos ao longo da BR 364 forneceram listas de serpentes para o estado de Rondônia (Vanzolini 1986, Nascimento et al. 1988).

Durante o resgate de fauna no enchimento do lago da Usina Hidrelétrica de Samuel em Candeias do Jamari, 1.016 serpentes pertencentes a 68 espécies foram obtidas (Jorge-da-Silva 1993). Jorgeda-Silva (1993) também compilou uma lista completa das serpentes registradas para o estado através de levantamento bibliográfico e consultas a algumas coleções científicas. Além dessas contribuições para o conhecimento das serpentes de Rondônia, outros estudos sobre comunidade ou levantamento de espécies foram desenvolvidos por Yuki et al. (1999), Brandão (2002), Bernarde \& Abe (2006), Turci \& Bernarde (2008) e Avila-Pires et al. (2009).

Devido ao aumento de estudos herpetofaunísticos em Rondônia e as mudanças taxonômicas recentes, a lista publicada por Jorgeda-Silva (1993) tornou-se desatualizada. Esse estudo teve como objetivo atualizar a lista de serpentes do estado de Rondônia a partir de levantamento bibliográfico e fornecer algumas informações sobre a distribuição das espécies no estado.

\section{Material e Métodos}

O estado de Rondônia, situado na região noroeste do Brasil (Figura 1), localiza-se entre os paralelos $7^{\circ} 58^{\prime}$ e $13^{\circ} 43^{\prime} \mathrm{S}$ e os meridianos $59^{\circ} 50^{\prime}$ e $66^{\circ} 48^{\prime} \mathrm{W}$, fazendo divisa ao norte com o estado do Amazonas, ao sul com a Bolívia, ao leste com o estado do Mato Grosso e ao oeste com a Bolívia e o estado do Acre (Oliveira 2002). A vegetação de Rondônia é caracterizada por um mosaico de áreas de floresta amazônica e de cerrado (Vanzolini 1986, Oliveira 2002), com predomínio de floresta (principalmente de Floresta Ombrófila Aberta).

Rondônia é constituído por 52 municípios divididos em oito microrregiões: Porto Velho (Buritis, Campo Novo de Rondônia, Candeias do Jamari, Cujubim, Itapuã do Oeste, Nova Mamoré e Porto Velho), Ariquemes (Alto Paraíso, Ariquemes, Cacaulândia, Machadinho d'Oeste, Monte Negro, Rio Crespo e Vale do Anari), Guajará-Mirim (Costa Marques, Guajará-Mirim e São Francisco do Guaporé), Alvorada d'Oeste (Alvorada d'Oeste, Nova Brasilândia d'Oeste, São Miguel do Guaporé e Seringueiras), Ji-Paraná (Governador Jorge Teixeira, Jaru, Ji-Paraná, Mirante da Serra, Nova União, Ouro Preto do Oeste, Presidente Médici, Teixeirópolis, Theobroma, Urupá e Vale do Paraíso), Cacoal (Alto Alegre dos Parecis, Alta Floresta d'Oeste, Cacoal, Castanheiras, Espigão do Oeste, Ministro Andreazza, Novo Horizonte do Oeste, Rolim de Moura e Santa Luzia d'Oeste), Colorado do Oeste (Cabixi, Cerejeiras, Colorado do Oeste, Corumbiara e Pimenteiras do Oeste) e Vilhena (Chupinguaia, Parecis, Pimenta Bueno, Primavera de Rondônia, São Felipe d'Oeste e Vilhena).

O presente trabalho foi baseado em informações disponíveis em literatura publicada sobre serpentes de Rondônia, como artigos de levantamento de espécies, estudos sobre ecologia, descrição de espécies, livros e notas sobre história natural e distribuição geográfica.

São apresentadas também imagens de várias espécies, sendo utilizado o registro fotográfico para documentar a ocorrência de espécies em algumas microrregiões no estado. Quando na ausência de imagens em vida de indivíduos procedentes de Rondônia, imagens de espécimes de outros estados foram apresentadas. Foram colocadas imagens adicionais de algumas espécies que apresentam mudança ontogenética de coloração ou policromatismo. As imagens utilizadas são dos autores e as respectivas autorias estão representadas pelas siglas dos nomes dos mesmos nas legendas: $\mathrm{PB}=$ Paulo Sérgio Bernarde, $\mathrm{SA}=$ Saymon de Albuquerque, $\mathrm{TB}=$ Thiago Oliveira Barros e LT $=$ Luiz Carlos Batista Turci. A relação de nomes populares de algumas espécies de serpentes foi baseada em Cunha \& Nascimento (1993), Marçal et al. (2011) e Bernarde et al. (2012).

Neste trabalho é seguida a nova proposta de classificação apresentada por Adalsteinsson et al. (2009), Carrasco et al. (2012) e Grazziotin et al. (2012).

\section{Resultados}

São registradas para o estado de Rondônia um total de 118 espécies de serpentes (Tabela 1; Figuras 2-14), pertencentes a oito famílias: Leptotyphlopidae (4 espécies), Typhlopidae (1), Aniliidae (1), Boidae (6), Colubridae (21), Dipsadidae (67), Elapidae (9) e Viperidae (9). Dessas, 109 foram registradas para áreas de floresta amazônica e 27 em cerrado. As microrregiões com maior número de espécies registradas foram a de Porto Velho (95 espécies), Cacoal (63) e Guajará-Mirim (55), enquanto que as com menor riqueza foram Ariquemes (18), Colorado do Oeste (3) e Alvorada d'Oeste (1) (Tabela 1; Figura 15). Dez espécies (Boa constrictor, Epicrates cenchria, Chironius exoletus, Drymarchon corais, Dipsas catesbyi, Leptodeira annulata, Liophis reginae, Oxyrhopus melanogenys, Xenopholis scalaris e Bothrops atrox) foram consideradas abundantes (mais de 30 registros), oito muito frequentes (entre 21 e 30), 48 frequentes (entre 6 e 20) e 52 pouco frequentes (menos de 5) (Tabela 1). 


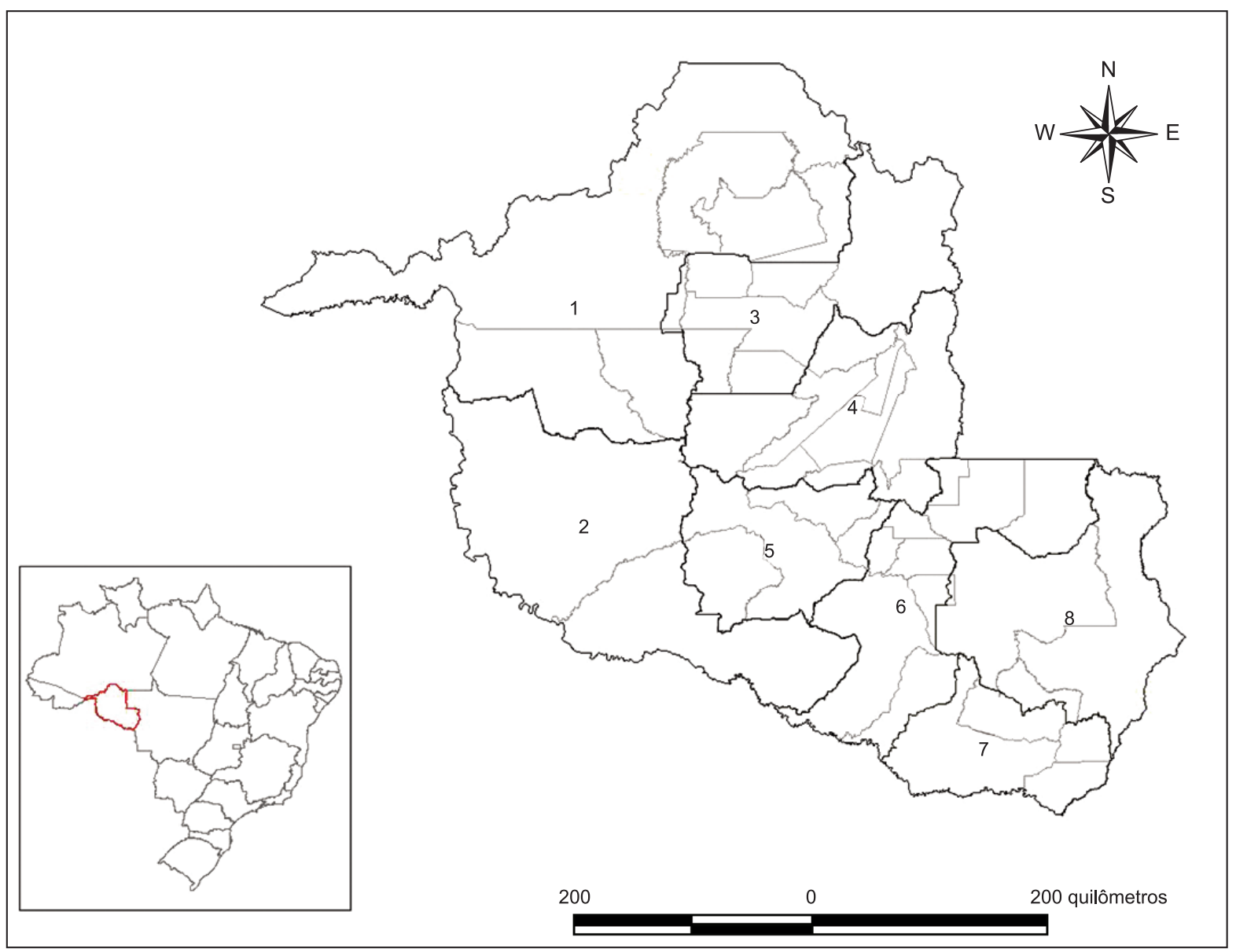

Figura 1. Microrregiões do estado de Rondônia (Brasil): $1=$ Porto Velho; $2=$ Guajará-Mirim; $3=$ Ariquemes; $4=$ Ji-paraná; $5=$ Alvorada d'Oeste; $6=$ Cacoal; $7=$ Colorado do Oeste; 8 = Vilhena.

Figure 1. Microregions of Rondônia State (Brazil): 1 = Porto Velho; 2 = Guajará-Mirim; 3 = Ariquemes; 4 = Ji-paraná; $5=$ Alvorada d'Oeste; $6=$ Cacoal; 7 = Colorado do Oeste; $8=$ Vilhena.

Tabela 1. Ocorrência das espécies de serpentes de Rondônia nas principais formações vegetais, sua frequência e distribuição nas microrregiões (PV = Porto Velho, $\mathrm{AR}=$ Ariquemes, $\mathrm{GM}=$ Guajará-Mirim, $\mathrm{AO}=$ Alvorada d'Oeste, $\mathrm{JP}=\mathrm{Ji}$ - Paraná, $\mathrm{CA}=\mathrm{Cacoal}, \mathrm{CO}=$ Colorado do Oeste e VI $=\mathrm{Vilhena}$ ). FREQ $=\mathrm{Frequência}$, $\mathrm{AB}=$ abundante (mais de 30 indivíduos registrados), $\mathrm{MF}=$ muito frequente (entre 21 e 30 indivíduos registrados), $\mathrm{FR}=$ freqüente (entre 6 a 20 indivíduos registrados), $\mathrm{PF}=$ pouco frequente (menos de 5 indivíduos registrados). Os números correspondem a referência bibliográfica de registro.

Table 1. Occurrence of snakes species in the main vegetation types of the state of Rondônia, their frequency and distribution in the microregions (PV $=$ Porto Velho, $\mathrm{AR}=$ Ariquemes, $\mathrm{GM}=$ Guajará-Mirim, $\mathrm{AO}=$ Alvorada d'Oeste, $\mathrm{JP}=\mathrm{Ji}$ - Paraná, $\mathrm{CA}=$ Cacoal, $\mathrm{CO}=$ Colorado do Oeste e VI $=\mathrm{Vilhena}) . \mathrm{FREQ}=$ Frequency, $\mathrm{AB}=$ abundant (more than 30 individuals recorded), $\mathrm{MF}=$ very frequent (between 21 and 30 individuals recorded), $\mathrm{FR}=$ frequent (between 6 and 20 individuals recorded), PF = infrequent (less than 5 individuals recorded). Numbers correspond to the bibliographic reference of record.

\begin{tabular}{|c|c|c|c|c|c|c|c|c|c|c|c|}
\hline Espécies $\Downarrow$ & Floresta Amazônica & Cerrado & FREQ & $\mathbf{P V}$ & $\mathbf{A R}$ & GM & AO & $\mathbf{J P}$ & $\mathbf{C A}$ & $\mathrm{CO}$ & VI \\
\hline \multicolumn{12}{|l|}{ Leptotyphlopidae (4) } \\
\hline Epictia diaplocia & 2,5 & & $\mathrm{PF}$ & + & & & & + & & & \\
\hline Epictia tenella & 3 & & $\mathrm{PF}$ & & & & & & + & & \\
\hline Siagonodon septemstriatus & 2 & & $\mathrm{PF}$ & + & & & & + & + & & \\
\hline Tricheilostoma macrolepis & 2,4 & & FR & + & & & & + & + & & \\
\hline
\end{tabular}

1. Esse estudo; 2. Jorge-da-Silva (1993); 3. Bernarde \& Abe (2006); 4. Vanzolini (1986); 5. Nascimento et al. (1988); 6. Avila-Pires et al. (2009); 7. Yuki et al. (1999); 8. Amaral (1948); 9. Brandão (2002); 10. França et al. (2006); 11. Turci \& Bernarde (2008); 12. Turci \& Bernarde (2009); 13. Albuquerque \& Freitas (2011); 14. Albuquerque \& Lema (2008); 15. Albuquerque et al. (2007); 16. Bailey et al. (2005); 17. Bernarde \& Moura-Leite (1999); 18. Bernarde et al. (2008); 19. Feitosa et al. (2007); 20. Fernandes et al. (2002); 21. Fernandes et al. (2004); 22. Fernandes et al. (2010); 23. Franco et al. (2007); 24. Frota (2005); 25. Gusmão et al. (2011); 26. Henderson (1997); 27. Henderson et al. (2009); 28. Hoge \& Nina (1964); 29. Keiser (1989); 30. Lema (2004); 31. Lema \& Albuquerque (2010); 32. Lema \& Renner (1998); 33. Lema et al. (2005); 34. Lima \& Prudente (2009); 35. Macedo-Bernarde (2006); 36. MacedoBernarde \& Bernarde (2005); 37. Machado (1993); 38. Moura-Leite \& Bernarde (1999); 39. Nascimento et al. (2008); 40. Passos \& Fernandes (2005); 41. Passos \& Fernandes (2009); 42. Prudente et al. (1998); 43. Prudente et al. (2008); 44. Roze \& Jorge-da-Silva (1990); 45. Santos-Junior. et al. (2008); 46. Scartozzoni et al. (2010); 47. Xavier-da-Silva \& Rodrigues (2008); 48. Whittaker (1996); 49. Zaher (1996); 50. Zaher et al. (2005); 51. Zaher et al. (2008); 52. Marçal et al. (2011); 53. Dixon et al. (1993); 54. Curcio et al. (2011); 55. Passos et al. (2004); 56. Griffin (1916); 57. Bernarde et al. (2011b); 58. Bernarde et al. (2012). 
Tabela 1. Continuação...

\begin{tabular}{|c|c|c|c|c|c|c|c|c|c|c|c|}
\hline Espécies $\Downarrow$ & Floresta Amazônica & Cerrado & FREQ & PV & $\mathbf{A R}$ & GM & AO & $\mathbf{J P}$ & $\mathbf{C A}$ & $\mathrm{CO}$ & VI \\
\hline \multicolumn{12}{|l|}{ Typhlopidae (1) } \\
\hline Typhlops reticulatus & $1,2,3,5,11,52$ & 10 & MF & + & & & & + & + & & + \\
\hline \multicolumn{12}{|l|}{ Aniliidae (1) } \\
\hline Anilius scytale & $1,2,3,4,11,12,52$ & & FR & + & & & & + & + & & \\
\hline \multicolumn{12}{|l|}{ Boidae (6) } \\
\hline Boa constrictor & $1,2,3,9,11,12,52$ & 10 & $\mathrm{AB}$ & + & & + & & + & + & & + \\
\hline Corallus batesii & $2,3,4,6,7,11,26,52$ & 2,26 & FR & + & & + & & + & + & & + \\
\hline Corallus hortulanus & $2,3,4,5,6,7,9,24,52$ & & MF & + & + & + & & + & + & & + \\
\hline Epicrates cenchria & $2,3,4,5,6,11,12,41,52$ & & $\mathrm{AB}$ & + & + & + & & & + & & \\
\hline Epicrates crassus & & 2,41 & PF & & & & & & & & + \\
\hline Eunectes murinus & $1,2,3,8,9,35,52$ & & FR & + & & + & & & + & & \\
\hline \multicolumn{12}{|l|}{ Colubridae (21) } \\
\hline Chironius carinatus & 52 & & $\mathrm{PF}$ & + & & & & & & & \\
\hline Chironius exoletus & $2,3,4,5,7,11,25,53$ & & $\mathrm{AB}$ & + & + & & + & & + & & + \\
\hline Chironius flavolineatus & & 13 & $\mathrm{PF}$ & & & & & & & & + \\
\hline Chironius fuscus & $2,4,5,6,7,52$ & & FR & + & & + & & + & & & + \\
\hline Chironius multiventris & $1,2,3,4,5,6,52$ & & FR & + & & + & & & + & & \\
\hline Chironius scurrulus & $1,2,3,4,5,6,7,52$ & & FR & + & & + & & + & + & & + \\
\hline Dendrophidion dendrophis & $1,2,3,4,6$ & & MF & + & & + & & & + & & \\
\hline Drymarchon corais & $2,3,4,5,11,12$ & 10 & $\mathrm{AB}$ & + & & + & & + & + & & + \\
\hline Drymobius rhombifer & 3,4 & & $\mathrm{PF}$ & & & & & & + & & \\
\hline Drymoluber brazili & & 10 & $\mathrm{PF}$ & & & & & & & & + \\
\hline Drymoluber dichrous & $2,3,4,5,6,52$ & & MF & + & & + & & & + & & \\
\hline Leptophis ahaetulla & $1,2,3,4,5,11,12,15,52$ & & MF & + & + & & & + & + & & \\
\hline Masticophis mentovarius & 3 & & $\mathrm{PF}$ & & & & & & + & & \\
\hline Mastigodryas boddaerti & $2,3,4,5,11,52$ & & FR & + & & & & & + & & \\
\hline Oxybelis aeneus & $1,2,4,52$ & 10 & FR & + & & & & & & & + \\
\hline Oxybelis fulgidus & $3,4,5,48,52$ & & FR & + & + & & & & + & & \\
\hline Pseustes poecilonotus & $1,2,3,4,6,52$ & & FR & + & & + & & + & + & & \\
\hline Pseustes sulphureus & $1,2,3,52$ & 2 & FR & + & & & & & + & & + \\
\hline Rhinobothryum lentiginosum & $1,2,3,6,11,52$ & 10 & FR & + & & + & & & + & & + \\
\hline Spilotes pullatus & $1,2,3,4,9,12,52$ & & FR & + & & + & & + & + & & \\
\hline Tantilla melanocephala & $1,2,3,4,6,52$ & 10 & FR & + & & + & & & + & & + \\
\hline \multicolumn{12}{|l|}{ Dipsadidae (67) } \\
\hline Apostolepis nigrolineata & $1,2,4,30,32,52,54$ & & $\mathrm{PF}$ & + & & & & & + & & \\
\hline Apostolepis quinquelineata & $1,30,32,54$ & & $\mathrm{PF}$ & + & & & & & & & \\
\hline Apostolepis striata & & 30 & $\mathrm{PF}$ & & & & & & & & + \\
\hline Atractus albuquerquei & $1,2,3,4,5,11,51,52$ & & MF & + & & & & + & + & & \\
\hline Atractus elaps & 2,4 & & $\mathrm{PF}$ & + & & & & & & & \\
\hline Atractus flammigerus & 2 & & $\mathrm{PF}$ & + & & & & & & & \\
\hline Atractus insipidus & 2 & & FR & + & & & & & & & \\
\hline
\end{tabular}

1. Esse estudo; 2. Jorge-da-Silva (1993); 3. Bernarde \& Abe (2006); 4. Vanzolini (1986); 5. Nascimento et al. (1988); 6. Avila-Pires et al. (2009); 7. Yuki et al. (1999); 8. Amaral (1948); 9. Brandão (2002); 10. França et al. (2006); 11. Turci \& Bernarde (2008); 12. Turci \& Bernarde (2009); 13. Albuquerque \& Freitas (2011); 14. Albuquerque \& Lema (2008); 15. Albuquerque et al. (2007); 16. Bailey et al. (2005); 17. Bernarde \& Moura-Leite (1999); 18. Bernarde et al. (2008); 19. Feitosa et al. (2007); 20. Fernandes et al. (2002); 21. Fernandes et al. (2004); 22. Fernandes et al. (2010); 23. Franco et al. (2007); 24. Frota (2005); 25. Gusmão et al. (2011); 26. Henderson (1997); 27. Henderson et al. (2009); 28. Hoge \& Nina (1964); 29. Keiser (1989); 30. Lema (2004); 31. Lema \& Albuquerque (2010); 32. Lema \& Renner (1998); 33. Lema et al. (2005); 34. Lima \& Prudente (2009); 35. Macedo-Bernarde (2006); 36. MacedoBernarde \& Bernarde (2005); 37. Machado (1993); 38. Moura-Leite \& Bernarde (1999); 39. Nascimento et al. (2008); 40. Passos \& Fernandes (2005); 41. Passos \& Fernandes (2009); 42. Prudente et al. (1998); 43. Prudente et al. (2008); 44. Roze \& Jorge-da-Silva (1990); 45. Santos-Junior. et al. (2008); 46. Scartozzoni et al. (2010); 47. Xavier-da-Silva \& Rodrigues (2008); 48. Whittaker (1996); 49. Zaher (1996); 50. Zaher et al. (2005); 51. Zaher et al. (2008); 52. Marçal et al. (2011); 53. Dixon et al. (1993); 54. Curcio et al. (2011); 55. Passos et al. (2004); 56. Griffin (1916); 57. Bernarde et al. (2011b); 58. Bernarde et al. (2012). 
Tabela 1. Continuação...

\begin{tabular}{|c|c|c|c|c|c|c|c|c|c|c|c|}
\hline Espécies $\Downarrow$ & Floresta Amazônica & Cerrado & FREQ & $\mathbf{P V}$ & $\mathbf{A R}$ & GM & AO & JP & $\mathbf{C A}$ & $\mathrm{CO}$ & VI \\
\hline Atractus latifrons & $1,2,3,4,6$ & & FR & + & & + & & + & + & & \\
\hline Atractus major & 1,6 & & $\mathrm{PF}$ & + & & + & & & & & \\
\hline Atractus schach & 1,5 & & $\mathrm{PF}$ & + & & & & & & & \\
\hline Atractus snethlageae & $1,2,3,4,6,52$ & & FR & + & & + & & & + & & \\
\hline Atractus taeniatus & 2 & & $\mathrm{PF}$ & + & & & & & & & \\
\hline Clelia clelia & 6,52 & & $\mathrm{PF}$ & + & & + & & & & & \\
\hline Clelia plumbea & $1,2,3,4,49$ & 10 & FR & + & & & & & + & & + \\
\hline Dipsas catesbyi & $1,2,3,4,5,11,12,34,52$ & 10,34 & $\mathrm{AB}$ & + & + & + & & + & + & & + \\
\hline Dipsas indica & $1,2,3,4,52$ & & FR & + & & + & & & + & + & \\
\hline Dipsas pavonina & $3,4,34$ & & $\mathrm{PF}$ & & & & & & + & & \\
\hline Dipsas variegata & $6,22,55$ & & $\mathrm{PF}$ & + & & + & & & & & \\
\hline Drepanoides anomalus & $1,2,3,4,6,7,11,52$ & & FR & + & & + & & & + & & + \\
\hline Erythrolamprus aesculapii & $1,2,3,5,52$ & & FR & + & & & & & + & & + \\
\hline Erythrolamprus mimus & 4 & & $\mathrm{PF}$ & & + & & & + & + & & \\
\hline Helicops angulatus & $1,3,4,6,9,52$ & 10 & MF & + & + & + & & & + & & + \\
\hline Helicops hagmanni & $1,2,6$ & & $\mathrm{PF}$ & + & & + & & & & & \\
\hline Helicops leopardinus & $1,24,56,52$ & & $\mathrm{PF}$ & + & & + & & & & & \\
\hline Helicops polylepis & 2,56 & & $\mathrm{PF}$ & + & & + & & & & & \\
\hline Hydrodynastes bicinctus & 23 & & $\mathrm{PF}$ & & & & & + & & & \\
\hline Hydrodynastes gigas & $1,3,7,9,17,52$ & & FR & + & & + & & & + & & + \\
\hline Hydrops martii & 9,52 & & $\mathrm{PF}$ & + & & + & & & & & \\
\hline Hydrops triangularis & 4,14 & & $\mathrm{PF}$ & + & & + & & & & & \\
\hline Imantodes cenchoa & $1,2,3,4,6,7,52$ & & FR & + & + & + & & + & + & & + \\
\hline Imantodes lentiferus & 2,6 & & $\mathrm{PF}$ & + & & + & & & & & \\
\hline Leptodeira annulata & $1,2,3,4,6,7,11,52$ & 10 & $\mathrm{AB}$ & + & + & + & & + & + & & + \\
\hline Liophis almadensis & $2,3,11$ & 10 & FR & & & & & & + & & + \\
\hline Liophis longiventris & 4 & 10 & $\mathrm{PF}$ & & & & & & & & + \\
\hline Liophis miliaris & 4 & & $\mathrm{PF}$ & & & & & + & & & \\
\hline Liophis oligolepis & 2,5 & & FR & + & & & & & & & \\
\hline Liophis poecilogyrus & 2,52 & & $\mathrm{PF}$ & + & & & & & & & \\
\hline Liophis reginae & $1,2,3,4,5,6,11,12,52$ & & $\mathrm{AB}$ & + & + & + & & + & + & + & + \\
\hline Liophis typhlus & $1,2,6,52$ & & FR & + & & + & & & & & \\
\hline Liophis taeniogaster & $3,20,52$ & & FR & + & + & & & + & + & & \\
\hline Ninia hudsoni & 2,3 & & $\mathrm{PF}$ & + & & & & & + & & \\
\hline Oxyrhopus formosus & $1,2,6$ & & $\mathrm{PF}$ & + & & + & & & & & \\
\hline Oxyrhopus melanogenys & $1,2,3,4,5,9,11,52$ & 10 & $\mathrm{AB}$ & + & & + & & & + & & + \\
\hline Oxyrhopus occipitalis & 1,2 & & $\mathrm{PF}$ & + & & & & & & & \\
\hline Oxyrhopus petolarius & $2,3,4,6,52$ & & FR & + & & + & & & + & & \\
\hline Oxyrhopus rhombifer & & 2,4 & $\mathrm{PF}$ & & & & & & & & + \\
\hline Oxyrhopus vanidicus & 1,4 & & $\mathrm{PF}$ & & + & & & & + & & \\
\hline Phalotris nasutus & 32 & & $\mathrm{PF}$ & & & & & & + & & \\
\hline
\end{tabular}

1. Esse estudo; 2. Jorge-da-Silva (1993); 3. Bernarde \& Abe (2006); 4. Vanzolini (1986); 5. Nascimento et al. (1988); 6. Avila-Pires et al. (2009); 7. Yuki et al. (1999); 8. Amaral (1948); 9. Brandão (2002); 10. França et al. (2006); 11. Turci \& Bernarde (2008); 12. Turci \& Bernarde (2009); 13. Albuquerque \& Freitas (2011); 14. Albuquerque \& Lema (2008); 15. Albuquerque et al. (2007); 16. Bailey et al. (2005); 17. Bernarde \& Moura-Leite (1999); 18. Bernarde et al. (2008); 19. Feitosa et al. (2007); 20. Fernandes et al. (2002); 21. Fernandes et al. (2004); 22. Fernandes et al. (2010); 23. Franco et al. (2007); 24. Frota (2005); 25. Gusmão et al. (2011); 26. Henderson (1997); 27. Henderson et al. (2009); 28. Hoge \& Nina (1964); 29. Keiser (1989); 30. Lema (2004); 31. Lema \& Albuquerque (2010); 32. Lema \& Renner (1998); 33. Lema et al. (2005); 34. Lima \& Prudente (2009); 35. Macedo-Bernarde (2006); 36. MacedoBernarde \& Bernarde (2005); 37. Machado (1993); 38. Moura-Leite \& Bernarde (1999); 39. Nascimento et al. (2008); 40. Passos \& Fernandes (2005); 41. Passos \& Fernandes (2009); 42. Prudente et al. (1998); 43. Prudente et al. (2008); 44. Roze \& Jorge-da-Silva (1990); 45. Santos-Junior. et al. (2008); 46. Scartozzoni et al. (2010); 47. Xavier-da-Silva \& Rodrigues (2008); 48. Whittaker (1996); 49. Zaher (1996); 50. Zaher et al. (2005); 51. Zaher et al. (2008); 52. Marçal et al. (2011); 53. Dixon et al. (1993); 54. Curcio et al. (2011); 55. Passos et al. (2004); 56. Griffin (1916); 57. Bernarde et al. (2011b); 58. Bernarde et al. (2012). 
Tabela 1. Continuação...

\begin{tabular}{|c|c|c|c|c|c|c|c|c|c|c|c|}
\hline Espécies $\Downarrow$ & Floresta Amazônica & Cerrado & FREQ & PV & $\mathbf{A R}$ & GM & AO & $\mathbf{J P}$ & $\mathbf{C A}$ & $\mathrm{CO}$ & VI \\
\hline Philodryas argentea & $1,2,3,4,5,6,7,37,43,52$ & & FR & + & + & + & & + & + & & + \\
\hline Philodryas boulengeri & $29,43,52$ & & $\mathrm{PF}$ & + & & & & & & & \\
\hline Philodryas olfersii & $3,4,9,11,12,52$ & 10 & FR & + & & + & & & + & & + \\
\hline Philodryas viridissima & $1,2,6,52$ & & FR & + & & + & & + & & + & \\
\hline Pseudoboa coronata & $1,2,52$ & & FR & + & & & & & & & \\
\hline Pseudoboa martinsi & 51 & & $\mathrm{PF}$ & + & & + & & & & & \\
\hline Pseudoboa nigra & & 10 & $\mathrm{PF}$ & & + & + & & & & & \\
\hline Pseudoeryx plicatilis & $1,28,46,52$ & & $\mathrm{PF}$ & + & & + & & & + & & \\
\hline Siphlophis cervinus & $1,2,6,42,52$ & & FR & + & & + & & & & & \\
\hline Siphlophis compressus & $1,2,3,4,6,7,52$ & & FR & + & & + & & & + & & + \\
\hline Siphlophis worontzowi & $1,2,3,42$ & & FR & + & & & & & + & & \\
\hline Taeniophalus brevirostris & 2 & & $\mathrm{PF}$ & + & & & & & & & \\
\hline Taeniophalus occipitalis & $1,2,3,45$ & 10 & FR & + & & & & & + & & + \\
\hline Thamnodynastes lanei & 16 & & $\mathrm{PF}$ & & & + & & & & & \\
\hline Thamnodynastes pallidus & $1,2,7,52$ & & $\mathrm{PF}$ & + & & & & & & & + \\
\hline Xenodon merremii & & 38 & $\mathrm{PF}$ & & & & & & & & + \\
\hline Xenodon rabdocephalus & $1,2,3,4,10,52$ & & FR & + & & + & & & + & & \\
\hline Xenodon severus & $1,2,3,5,52$ & & FR & + & & & & & + & & \\
\hline Xenopholis scalaris & $1,2,3,4,6,52$ & & $\mathrm{AB}$ & + & + & + & & & + & & \\
\hline \multicolumn{12}{|l|}{ Elapidae (9) } \\
\hline Micrurus albicinctus & $1,2,6,44,52$ & & FR & + & & + & & & & & \\
\hline Micrurus hemprichii & $1,2,4,44,52$ & & FR & + & & & & & & & \\
\hline Micrurus lemniscatus & $1,2,4,5,6,11,44,52$ & & FR & + & & + & & + & + & & \\
\hline Micrurus mipartitus & 4 & & $\mathrm{PF}$ & + & & & & & & & \\
\hline Micrurus paraensis & $5,19,52$ & & $\mathrm{PF}$ & + & & & & + & & & \\
\hline Micrurus cf. ornatissimus & 1,52 & & $\mathrm{PF}$ & + & & & & + & & & \\
\hline Micrurus spixii & $1,2,3,4,5,11,12,44,52$ & & MF & + & + & & & & + & & \\
\hline Micrurus surinamensis & $1,2,3,4,6,11,40,52$ & & FR & + & + & + & & & + & & \\
\hline Micrurus sp. & 1 & & $\mathrm{PF}$ & & & & & & + & & + \\
\hline \multicolumn{12}{|l|}{ Viperidae (9) } \\
\hline Bothrocophias hyoprora & $1,2,52$ & & FR & + & & & & & & & \\
\hline $\begin{array}{l}\text { Bothrocophias } \\
\text { microphthalmus }\end{array}$ & 18,58 & & $\mathrm{PF}$ & & & & & + & & & \\
\hline Bothrops atrox & $1,2,3,4,5,6,7,37,40,52$ & 1 & $\mathrm{AB}$ & + & + & + & & + & + & & + \\
\hline Bothrops bilineata & $1,2,3,6,11,, 52,57$ & & FR & + & & + & & & + & & \\
\hline Bothrops brazili & $1,2,4,52$ & & FR & + & & & & & & & \\
\hline Bothrops mattogrossensis & & $2,4,10,47$ & $\mathrm{PF}$ & & & & & & & & + \\
\hline Bothrops taeniata & $1,2,6$ & & FR & + & & + & & & & & \\
\hline Crotalus durissus & & 2,10 & $\mathrm{PF}$ & & & & & & & & + \\
\hline Lachesis muta & $2,3,4,21$ & & FR & + & & & & & + & & \\
\hline Total de espécies & 109 & 27 & & 95 & 18 & 55 & 1 & 30 & 63 & 3 & 39 \\
\hline
\end{tabular}

1. Esse estudo; 2. Jorge-da-Silva (1993); 3. Bernarde \& Abe (2006); 4. Vanzolini (1986); 5. Nascimento et al. (1988); 6. Avila-Pires et al. (2009); 7. Yuki et al. (1999); 8. Amaral (1948); 9. Brandão (2002); 10. França et al. (2006); 11. Turci \& Bernarde (2008); 12. Turci \& Bernarde (2009); 13. Albuquerque \& Freitas (2011); 14. Albuquerque \& Lema (2008); 15. Albuquerque et al. (2007); 16. Bailey et al. (2005); 17. Bernarde \& Moura-Leite (1999); 18. Bernarde et al. (2008); 19. Feitosa et al. (2007); 20. Fernandes et al. (2002); 21. Fernandes et al. (2004); 22. Fernandes et al. (2010); 23. Franco et al. (2007); 24. Frota (2005); 25. Gusmão et al. (2011); 26. Henderson (1997); 27. Henderson et al. (2009); 28. Hoge \& Nina (1964); 29. Keiser (1989); 30. Lema (2004); 31. Lema \& Albuquerque (2010); 32. Lema \& Renner (1998); 33. Lema et al. (2005); 34. Lima \& Prudente (2009); 35. Macedo-Bernarde (2006); 36. MacedoBernarde \& Bernarde (2005); 37. Machado (1993); 38. Moura-Leite \& Bernarde (1999); 39. Nascimento et al. (2008); 40. Passos \& Fernandes (2005); 41. Passos \& Fernandes (2009); 42. Prudente et al. (1998); 43. Prudente et al. (2008); 44. Roze \& Jorge-da-Silva (1990); 45. Santos-Junior. et al. (2008); 46. Scartozzoni et al. (2010); 47. Xavier-da-Silva \& Rodrigues (2008); 48. Whittaker (1996); 49. Zaher (1996); 50. Zaher et al. (2005); 51. Zaher et al. (2008); 52. Marçal et al. (2011); 53. Dixon et al. (1993); 54. Curcio et al. (2011); 55. Passos et al. (2004); 56. Griffin (1916); 57. Bernarde et al. (2011b); 58. Bernarde et al. (2012). 

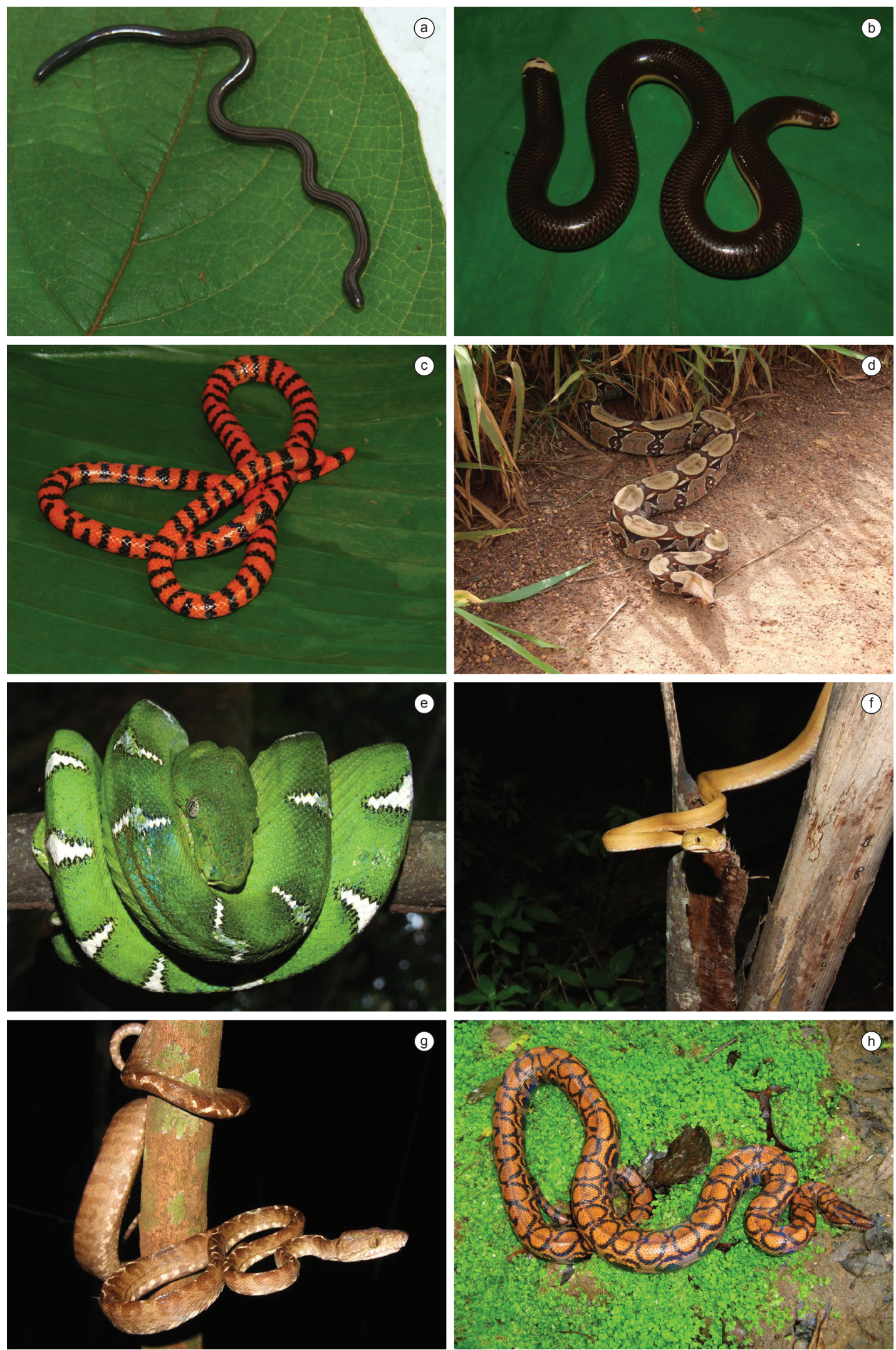

Figura 2. a) Epictia tenella - Espigão do Oeste (RO). Foto por PB; b) Typhlops reticulatus - Porto Velho (RO). LT; c) Anilius scytale - Porto Velho (RO). Fotos por LT; d) Boa constrictor - Espigão do Oeste (RO); e) Corallus batesii - AM; f) C. hortulanus - AM; g) C. hortulanus - MT. Fotos por PB; h) Epicrates cenchria - Porto Velho (RO). Foto por SA.

Figure 2. a) Epictia tenella - Espigão do Oeste (RO). Photo by PB; b) Typhlops reticulatus - Porto Velho (RO). LT; c) Anilius scytale - Porto Velho (RO). Photos by LT; d) Boa constrictor - Espigão do Oeste (RO); e) Corallus batesii - AM; f) C. hortulanus - AM; g) C. hortulanus - MT. Photos by PB; h) Epicrates cenchria - Porto Velho (RO). Photo by SA. 

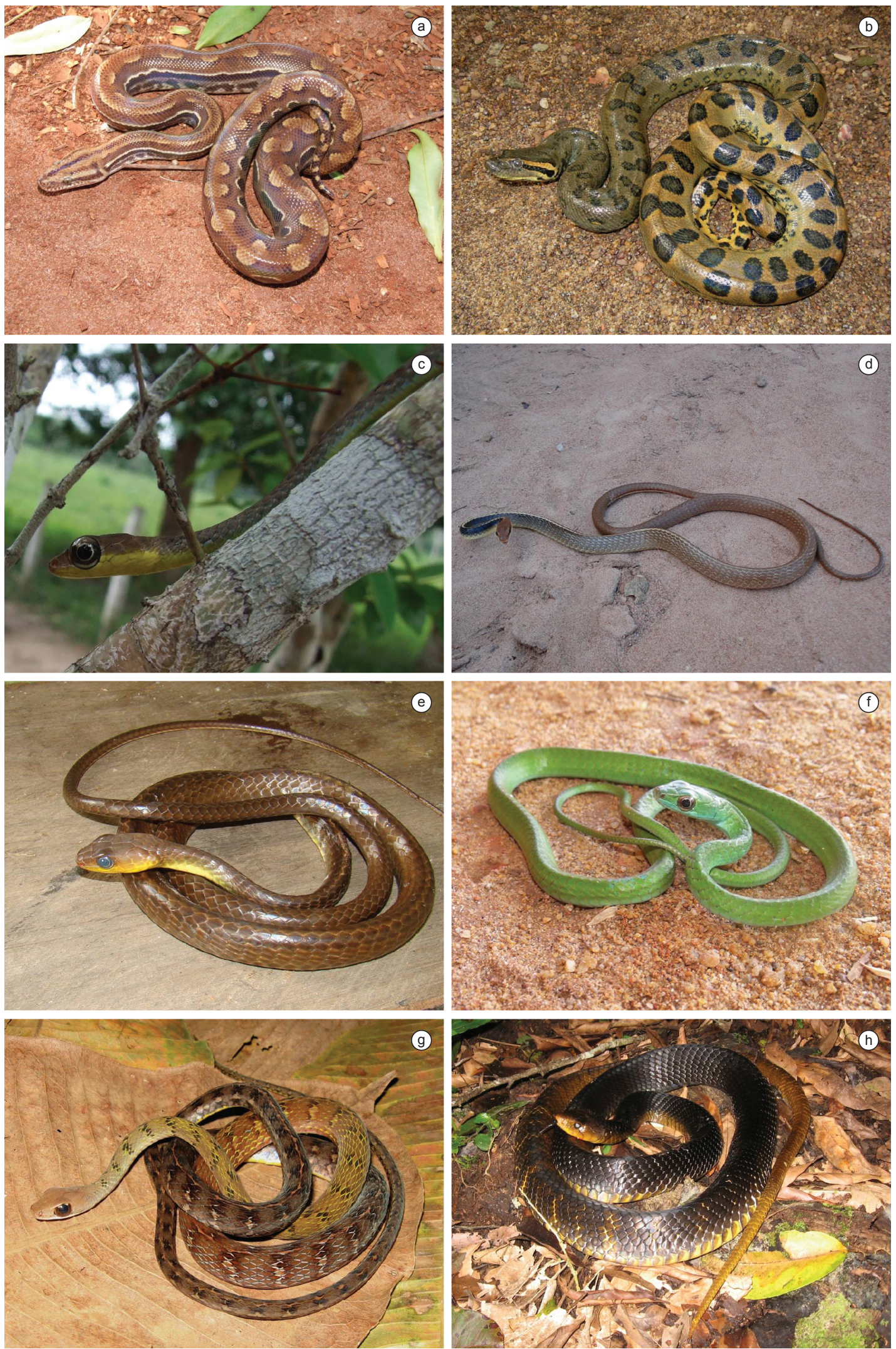

Figura 3. a) Epicrates crassus - MS. Foto por PB; b) Eunectes murinus - Porto Velho (RO). Foto por SA; c) Chironius exoletus - Machadinho do Oeste (RO). Foto por PB; d) C. flavolineatus - Pimenta Bueno (RO). Foto por SA; e) C. multiventris - Porto Velho (RO). Foto por TB; f) C. scurrulus (juvenil) - Porto Velho (RO); g) Dendrophidion dendrophis - Porto Velho (RO). Fotos por SA; h) Drymarchon corais - AC. Foto por PB.

Figure 3. a) Epicrates crassus - MS. Photo by PB; b) Eunectes murinus - Porto Velho (RO). Photo by SA; c) Chironius exoletus - Machadinho do Oeste (RO). Photo by PB; d) C. flavolineatus - Pimenta Bueno (RO). Photo by SA; e) C. multiventris - Porto Velho (RO). Photo by TB; f) C. scurrulus (juvenile) - Porto Velho (RO); g) Dendrophidion dendrophis - Porto Velho (RO). Photos by SA; h) Drymarchon corais - AC. Photo by PB. 

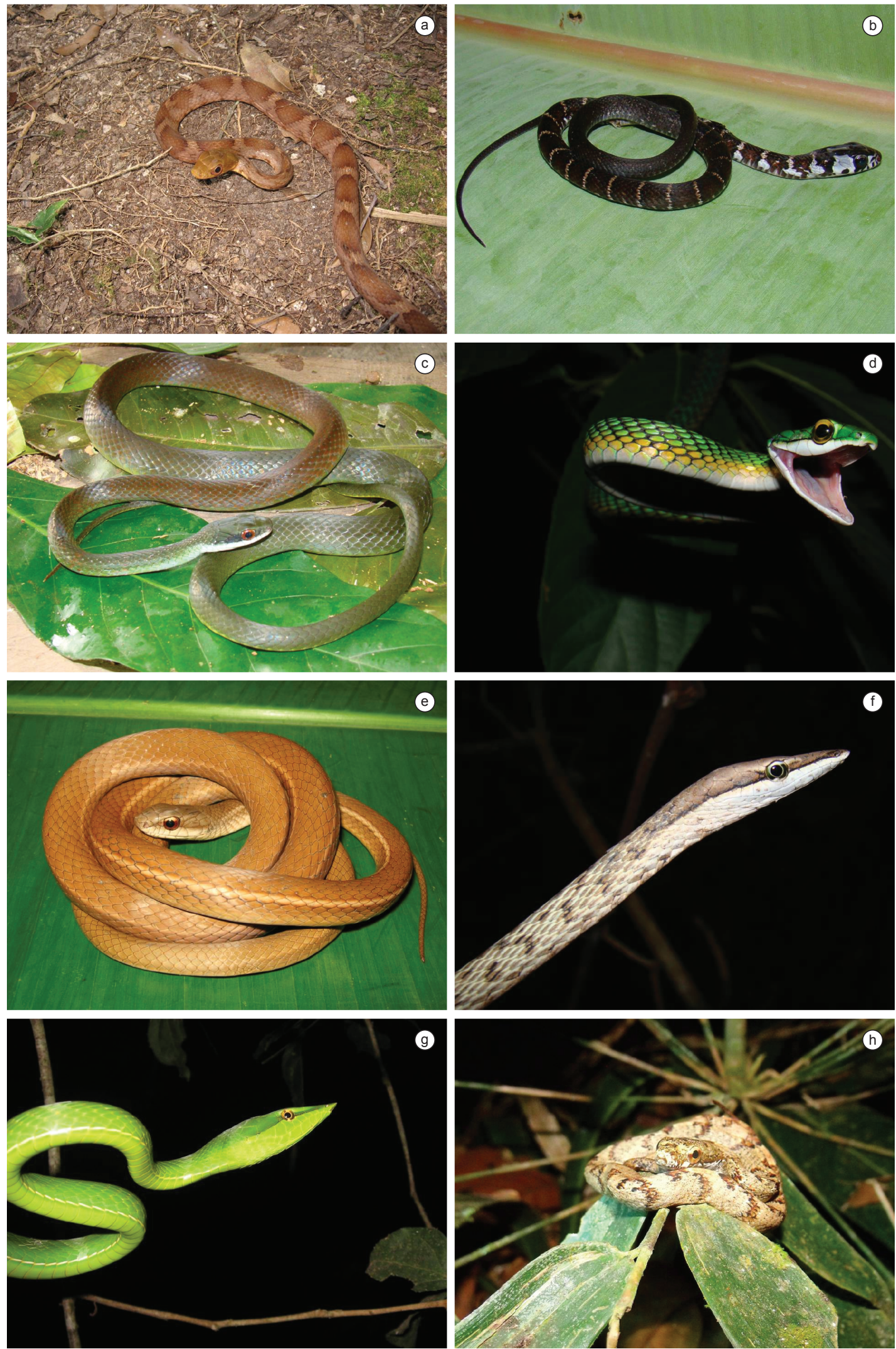

Figura 4. a) Drymobius rhombifer - AC. Foto por PB; b) Drymoluber dichrous (juvenil) - Porto Velho (RO); c) D. dichrous - Porto Velho (RO). Fotos por TB; d) Leptophis ahaetulla - Porto Velho (RO). Foto por LT; e) Mastigodryas boddaerti - Porto Velho (RO). Foto por SA; f) Oxybelis aeneus - Porto Velho (RO). Foto por LT; g) O. fulgidus - Espigão do Oeste (RO). Foto por PB; h) Pseustes poecilonotus (juvenil) - Porto Velho (RO). Foto por SA.

Figure 4. a) Drymobius rhombifer - AC. Photo by PB; b) Drymoluber dichrous (juvenile) - Porto Velho (RO); c) D. dichrous - Porto Velho (RO). Photos by TB; d) Leptophis ahaetulla - Porto Velho (RO). Photo by LT; e) Mastigodryas boddaerti - Porto Velho (RO). Photo by SA; f) Oxybelis aeneus - Porto Velho (RO). Photo by LT; g) O. fulgidus - Espigão do Oeste (RO). Photo by PB; h) Pseustes poecilonotus (juvenile) - Porto Velho (RO). Photo by SA. 

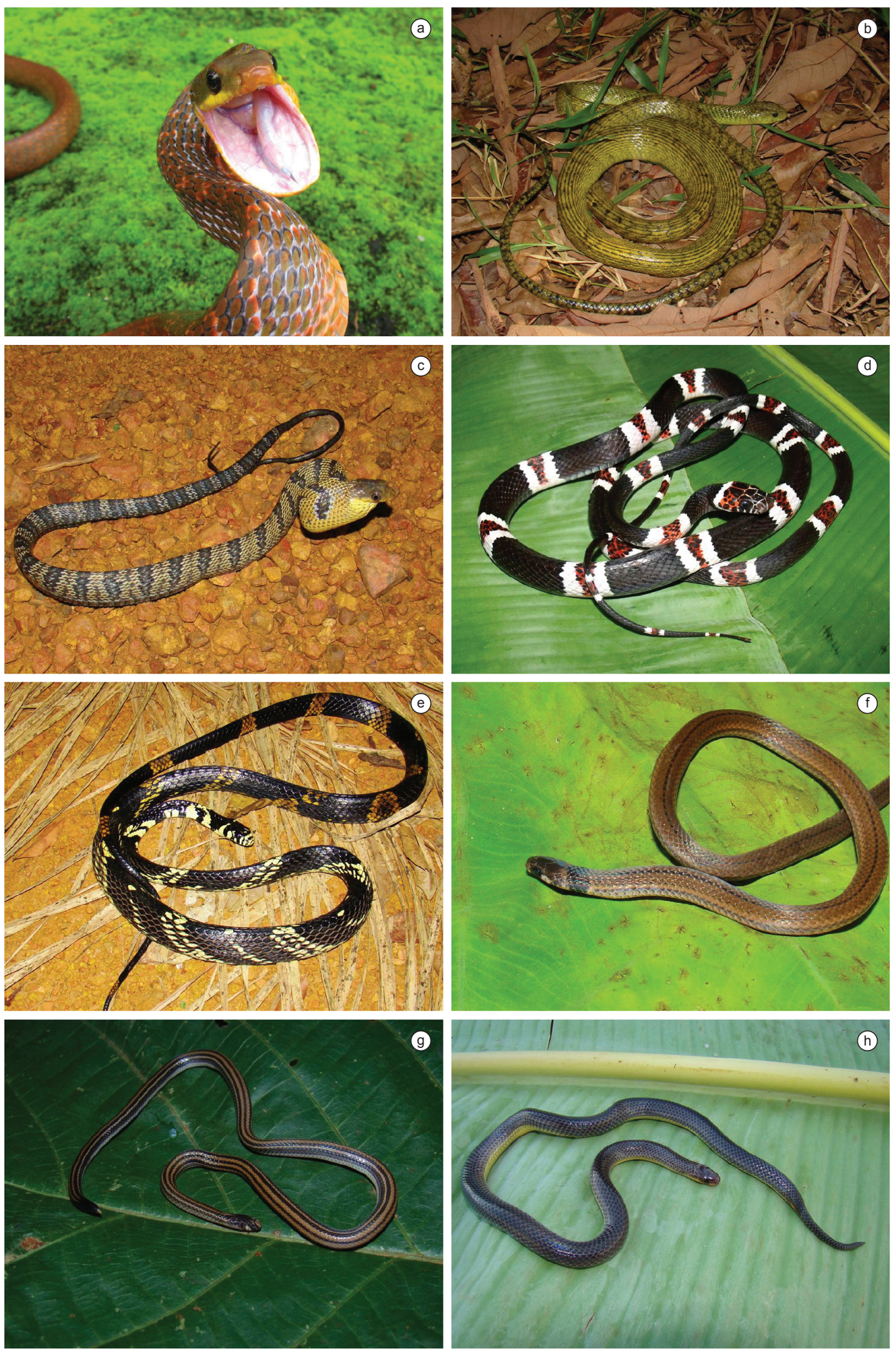

Figura 5. a) Pseustes poecilonotus - Candeias do Jamari (RO). Foto por SA; b) P. sulphureus - Porto Velho (RO). Foto por LT; c) P. sulphureus - Porto Velho (RO). Foto por TB; d) Rhinobothryum lentiginosum - Porto Velho (RO). Foto por SA; e) Spilotes pullatus - Porto Velho (RO); f) Tantilla melanocephala - Porto Velho (RO). Fotos por TB; g) Apostolepis nigrolineata - Porto Velho (RO). Foto por LT; h) Atractus albuquerquei - Porto Velho (RO). Foto por TB.

Figure 5. a) Pseustes poecilonotus - Candeias do Jamari (RO). Photo by SA; b) P. sulphureus - Porto Velho (RO). Photo by LT; c) P. sulphureus - Porto Velho (RO). Photo by TB; d) Rhinobothryum lentiginosum - Porto Velho (RO). Photo by SA; e) Spilotes pullatus - Porto Velho (RO); f) Tantilla melanocephala - Porto Velho (RO). Photos by TB; g) Apostolepis nigrolineata - Porto Velho (RO). Photo by LT; h) Atractus albuquerquei - Porto Velho (RO). Photo by TB. 

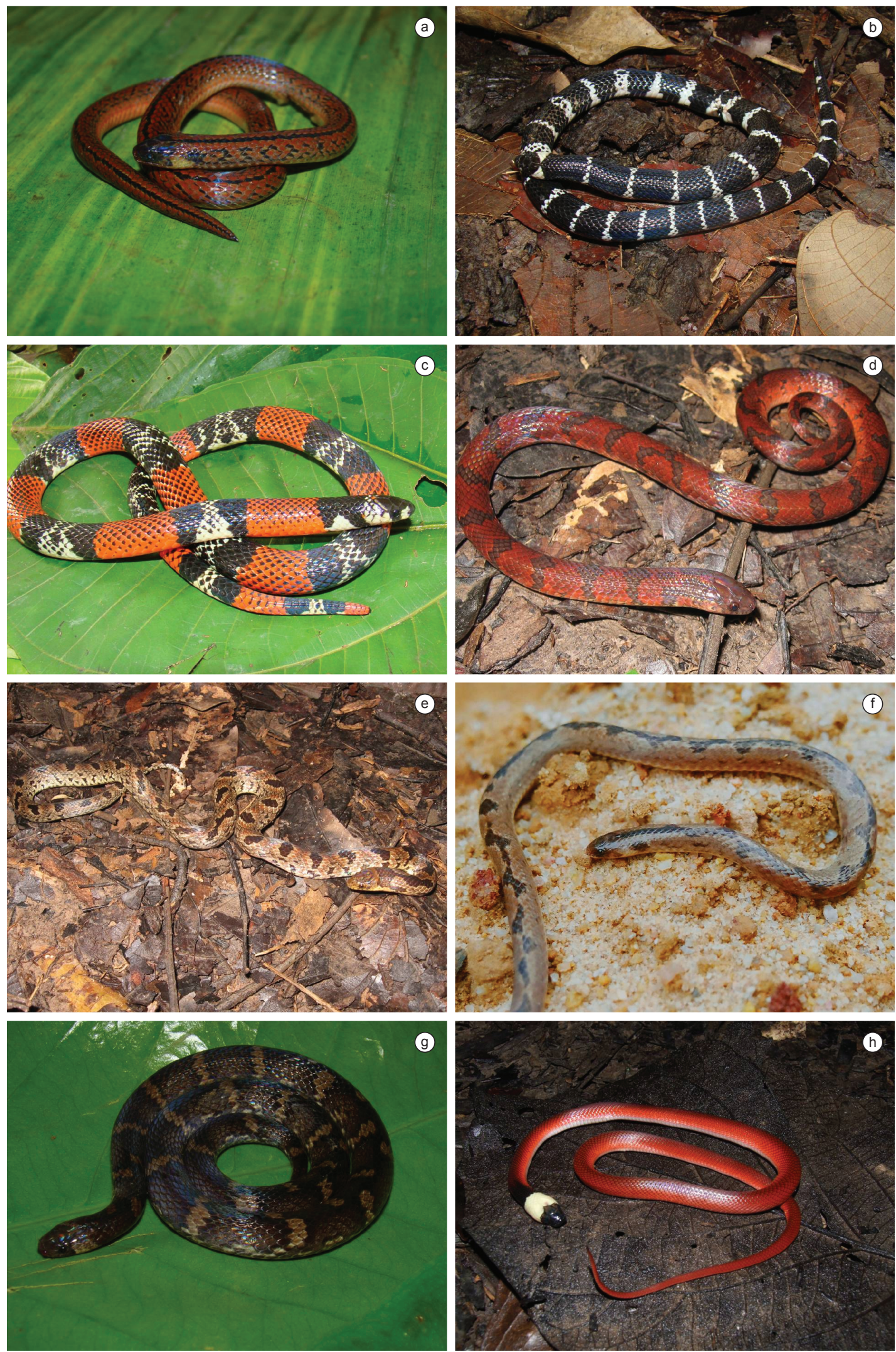

Figura 6. a) Atractus insipidus - Porto Velho (RO); b) A. latifrons - Porto Velho (RO). Fotos por LT; c) A. latifrons - Porto Velho (RO). Foto por TB; d) e e) A. major - AC. Fotos por PB; f) A. schach - Candeias do Jamari (RO). Foto por SA; g) A. snethlageae - Porto Velho (RO). Foto por LT; h) Clelia clelia (juvenil) - AC. Foto por PB.

Figure 6. a) Atractus insipidus - Porto Velho (RO); b) A. latifrons - Porto Velho (RO). Photos by LT; c) A. latifrons - Porto Velho (RO). Photo by TB; d) e e) A. major - AC. Photos by PB; f) A. schach - Candeias do Jamari (RO). Photo by SA; g) A. snethlageae - Porto Velho (RO). Photo by LT; h) Clelia clelia (juvenile) - AC. Photo by PB. 

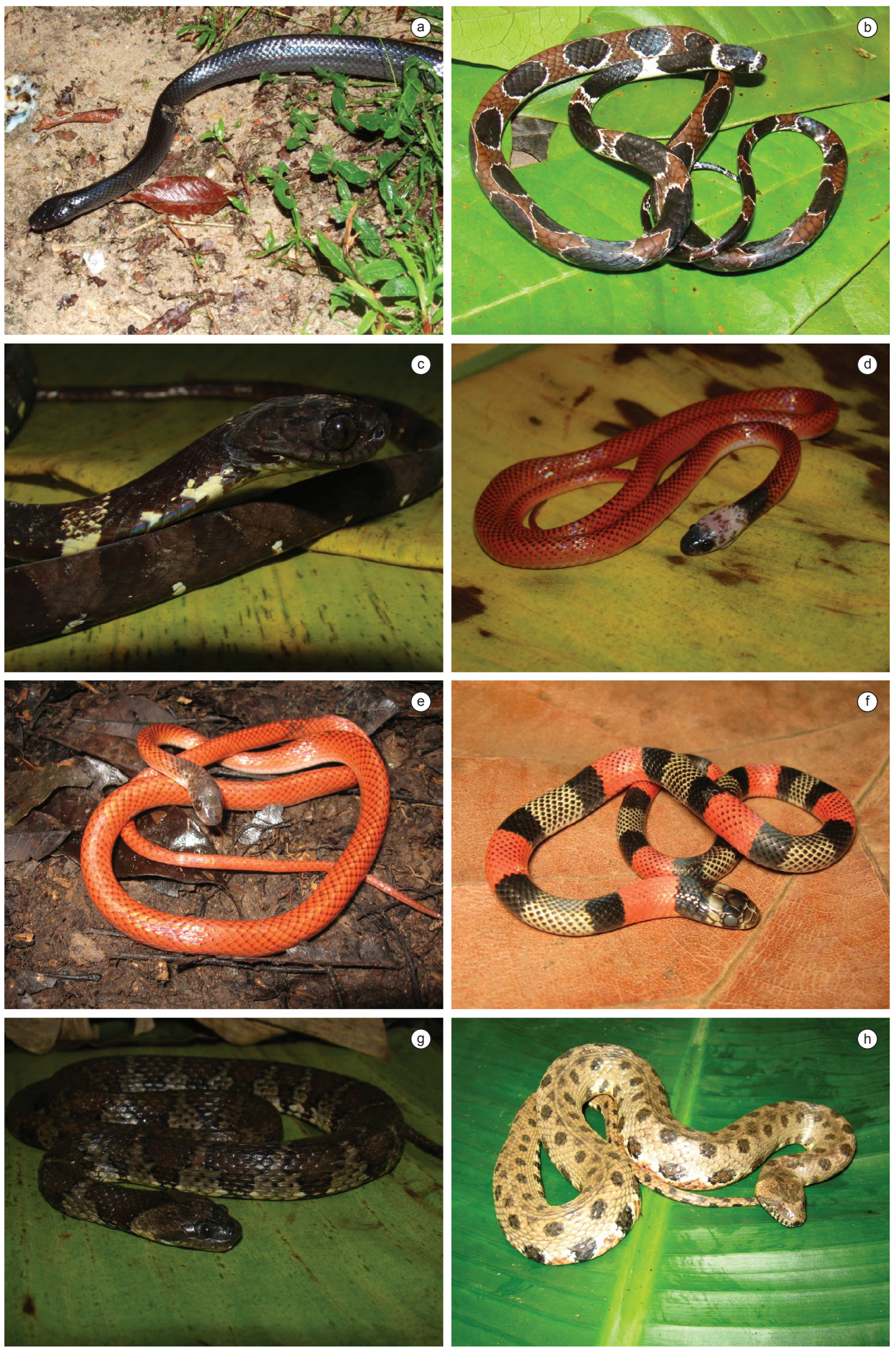

Figura 7. a) Clelia clelia - AC. Foto por PB; b) Dipsas catesbyi - Porto Velho (RO). Foto por TB; c) D. indica - Porto Velho (RO); d) Drepanoides anomalus - Porto Velho (RO). Fotos por LT; e) D. anomalus - AC. Foto por PB; f) Erythrolamprus aesculapii - Porto Velho (RO). Foto por SA; g) Helicops angulatus - Porto Velho (RO). Foto por LT; h) H. hagmanni - Porto Velho (RO). Foto por SA.

Figure 7. a) Clelia clelia - AC. Photo by PB; b) Dipsas catesbyi - Porto Velho (RO). Photo by TB; c) D. indica - Porto Velho (RO); d) Drepanoides anomalus - Porto Velho (RO). Photos by LT; e) D. anomalus - AC. Photo by PB; f) Erythrolamprus aesculapii - Porto Velho (RO). Photo by SA; g) Helicops angulatus - Porto Velho (RO). Photo by LT; h) H. hagmanni - Porto Velho (RO). Photo by SA. 

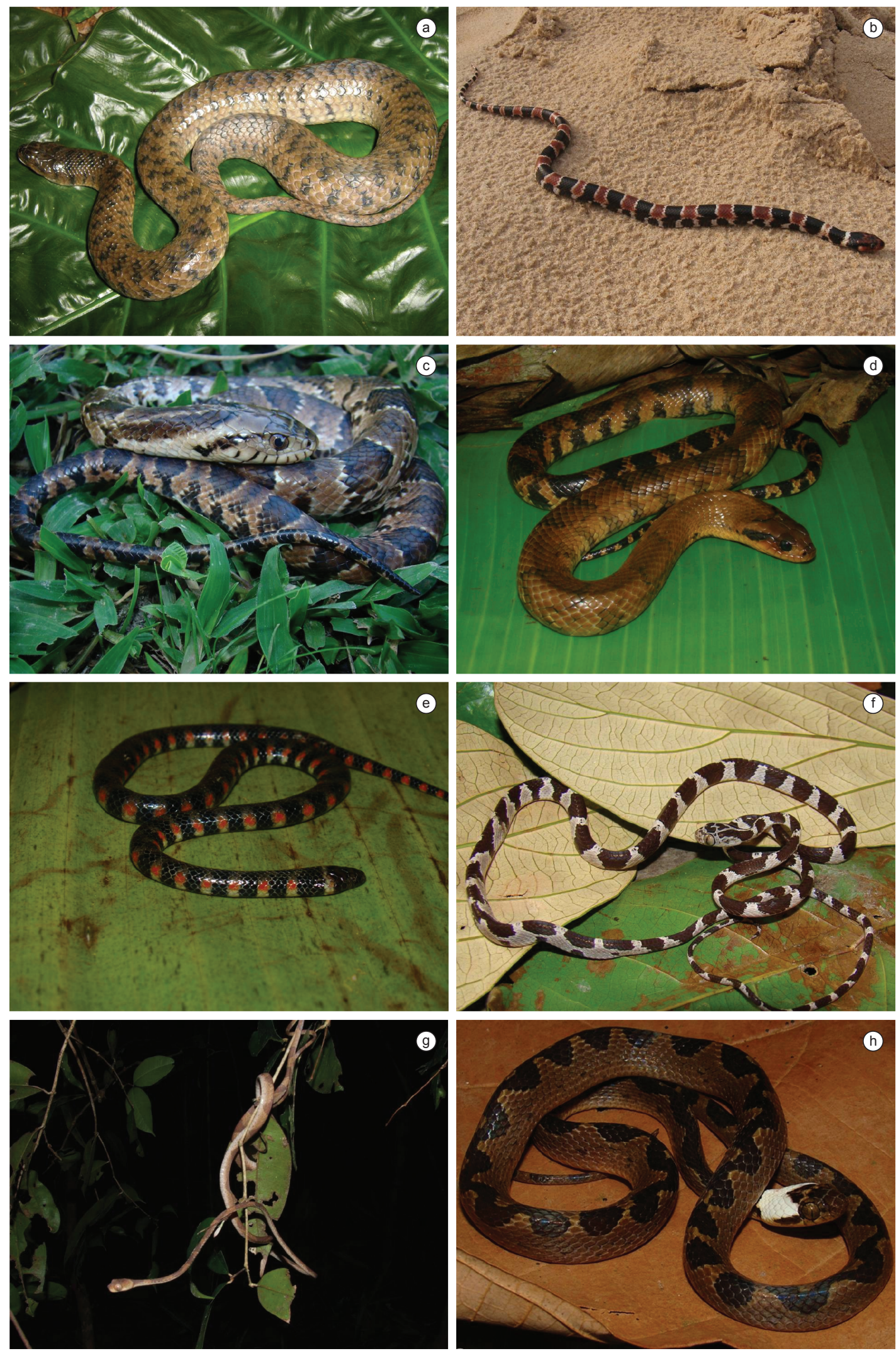

Figura 8. a) Helicops leopardinus - Porto Velho (RO). Foto por SA; b) Hydrodynastes bicinctus - MT. Foto por PB; c) e d) H. gigas - Porto Velho (RO); e) Hydrops triangularis - Porto Velho. Fotos por LT; f) Imantodes cenchoa - Porto Velho (RO). Foto por TB; g) I. lentiferus - AM. Foto por PB; h) Leptodeira annulata (juvenil) - Porto Velho (RO). Foto por LT.

Figure 8. a) Helicops leopardinus - Porto Velho (RO). Photo by SA; b) Hydrodynastes bicinctus - MT. Photo by PB; c) e d) H. gigas - Porto Velho (RO); e) Hydrops triangularis - Porto Velho. Photos by LT; f) Imantodes cenchoa - Porto Velho (RO). Photo by TB; g) I. lentiferus - AM. Photo by PB; h) Leptodeira annulata (juvenile) - Porto Velho (RO). Photo by LT. 

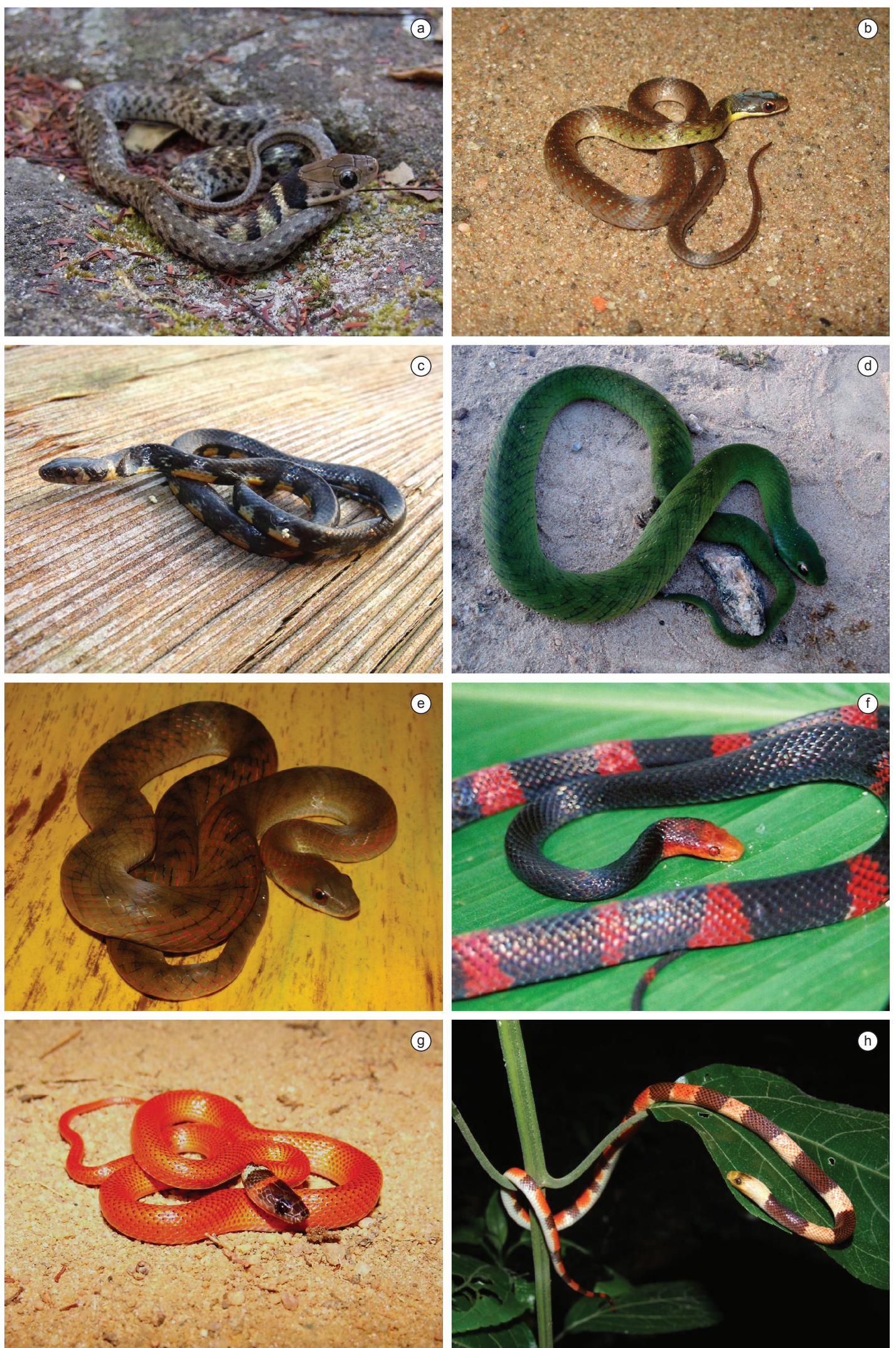

Figura 9. a) Liophis poecilogyrus (juvenil) - MS. Foto por PB; b) L. reginae - Monte Negro (RO); c) L. taeniogaster - Itapuã do Oeste (RO); d) Liophis typhlus - Pimenta Bueno (RO). Fotos por SA; e) L. typhlus - Porto Velho (RO). Foto por LT; f) Oxyrhopus formosus - Monte Negro (RO); g) O. melanogenys - Itapuã do Oeste (RO). Fotos por SA; h) O. occipitalis (juvenil) - AC. Foto por PB.

Figure 9. a) Liophis poecilogyrus (juvenile) - MS. Photo by PB; b) L. reginae - Monte Negro (RO); c) L. taeniogaster - Itapuã do Oeste (RO); d) Liophis typhlus - Pimenta Bueno (RO). Photos by SA; e) L. typhlus - Porto Velho (RO). Photo by LT; f) Oxyrhopus formosus - Monte Negro (RO); g) O. melanogenys - Itapuã do Oeste (RO). Photos by SA; h) O. occipitalis (juvenile) - AC. Photo by PB. 
Bernarde, P.S. et al.
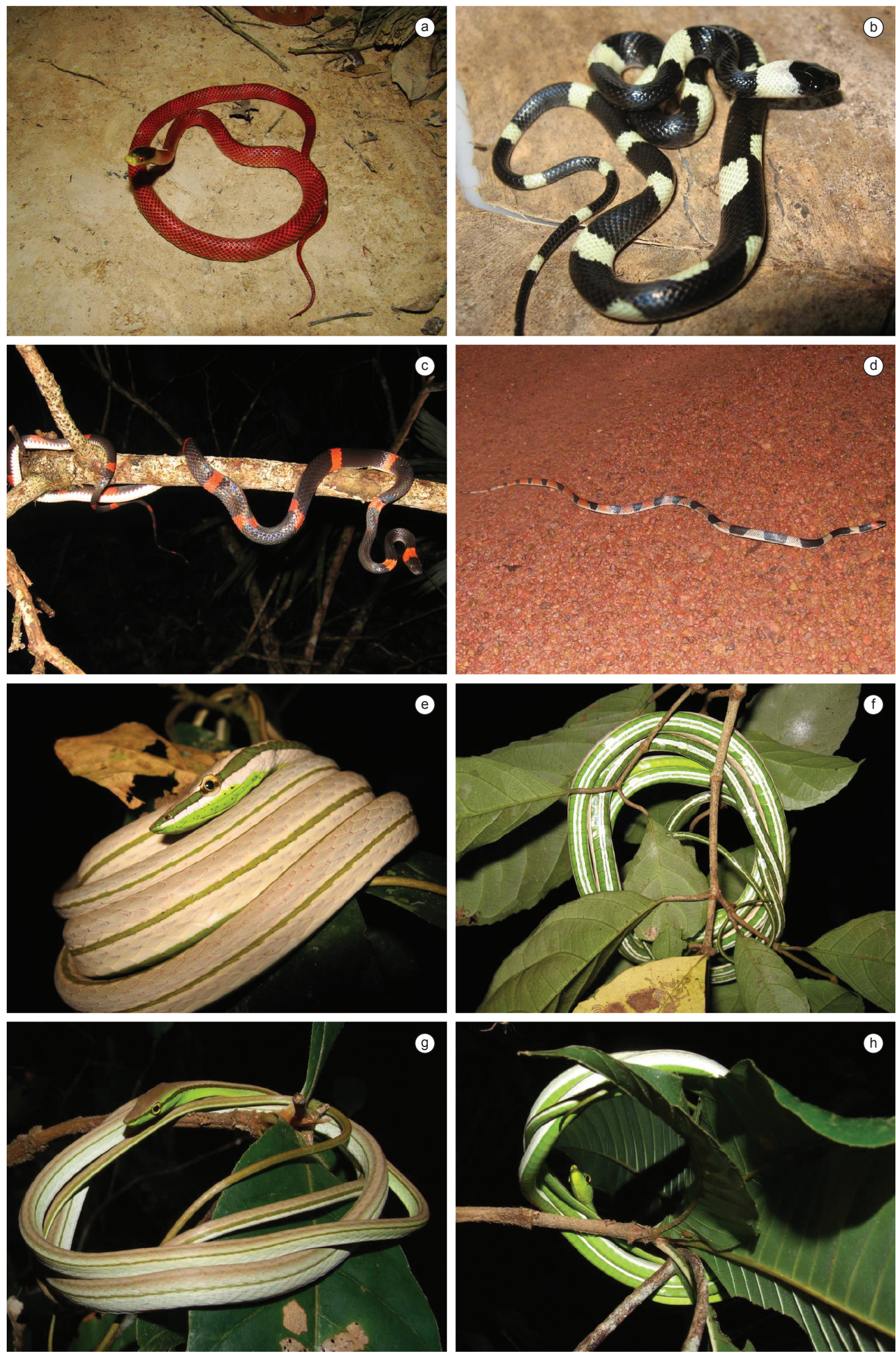

Figura 10. a) Oxyrhopus occipitalis - Porto Velho (RO). Foto por TB; b) O. petolarius (juvenil) - AC; c) O. petolarius - AC; d) O. rhombifer - AM; e) e f) Philodryas argentea - AC; g) e h) P. boulengeri - AM. Fotos por PB

Figure 10. a) Oxyrhopus occipitalis - Porto Velho (RO). Photo by TB; b) O. petolarius (juvenile) - AC; c) O. petolarius - AC; d) O. rhombifer - AM; e) e f) Philodryas argentea - AC; g) e h) P. boulengeri - AM. Photos by PB. 

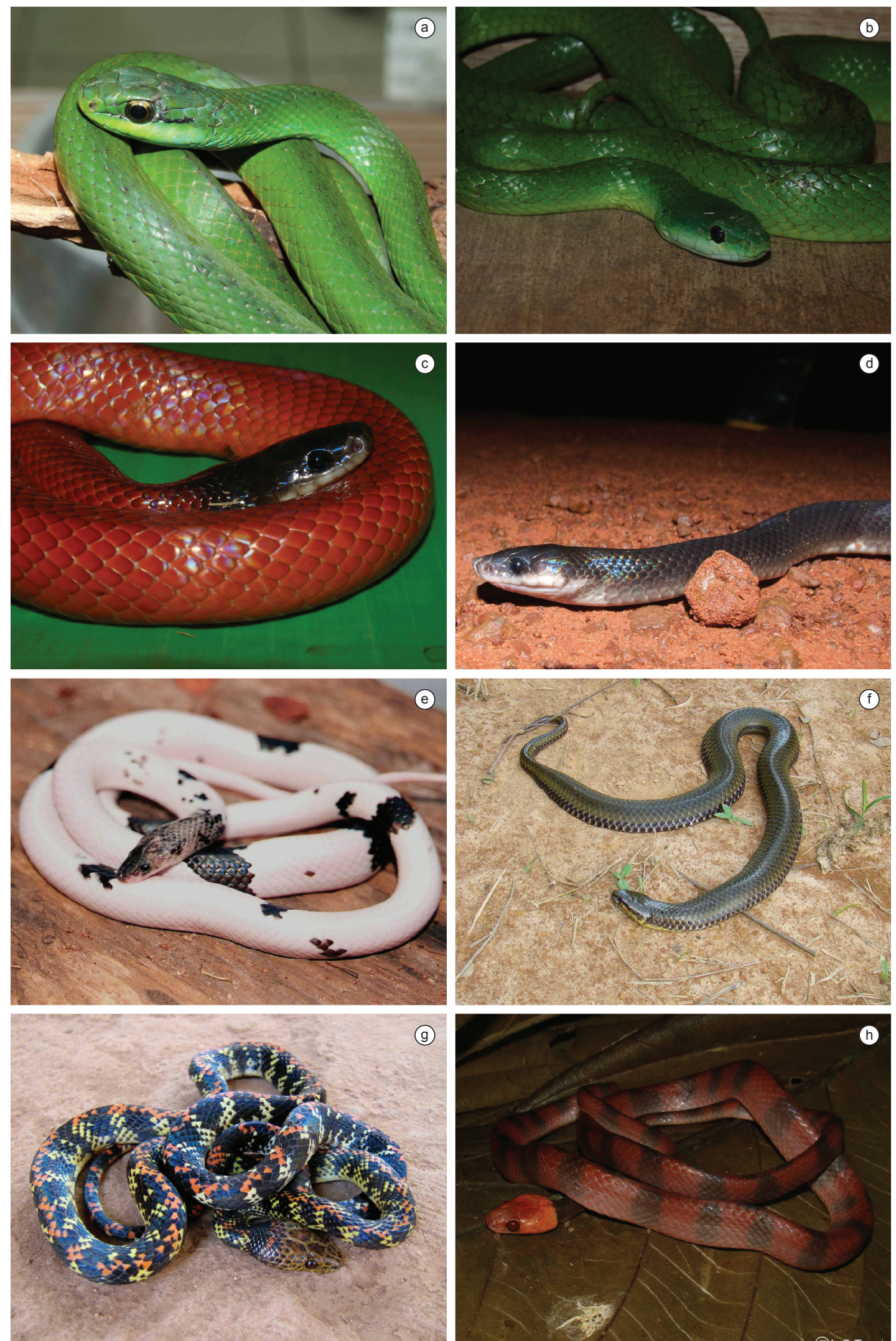

Figura 11. a) Philodryas olfersii - MS. Foto por PB; b) P. viridissima - Porto Velho (RO); c) P. coronata - Porto Velho (RO). Fotos por LT; d) P. nigra - AM. Foto por PB; e) P. nigra - Monte Negro (RO). Foto por SA; f) Pseudoeryx plicatilis - Porto Velho (RO). Foto por TB; g) Siphlophis cervinus - Porto Velho (RO). Foto por SA; h) S. compressus - Porto Velho (RO). Foto por LT.

Figure 11. a) Philodryas olfersii - MS. Photo by PB; b) P. viridissima - Porto Velho (RO); c) P. coronata - Porto Velho (RO). Photos by LT; d) P. nigra - AM. Photo by PB; e) P. nigra - Monte Negro (RO). Photo by SA; f) Pseudoeryx plicatilis - Porto Velho (RO). Photo by TB; g) Siphlophis cervinus - Porto Velho (RO). Photo by SA; h) S. compressus - Porto Velho (RO). Photo by LT. 
Bernarde, P.S. et al.
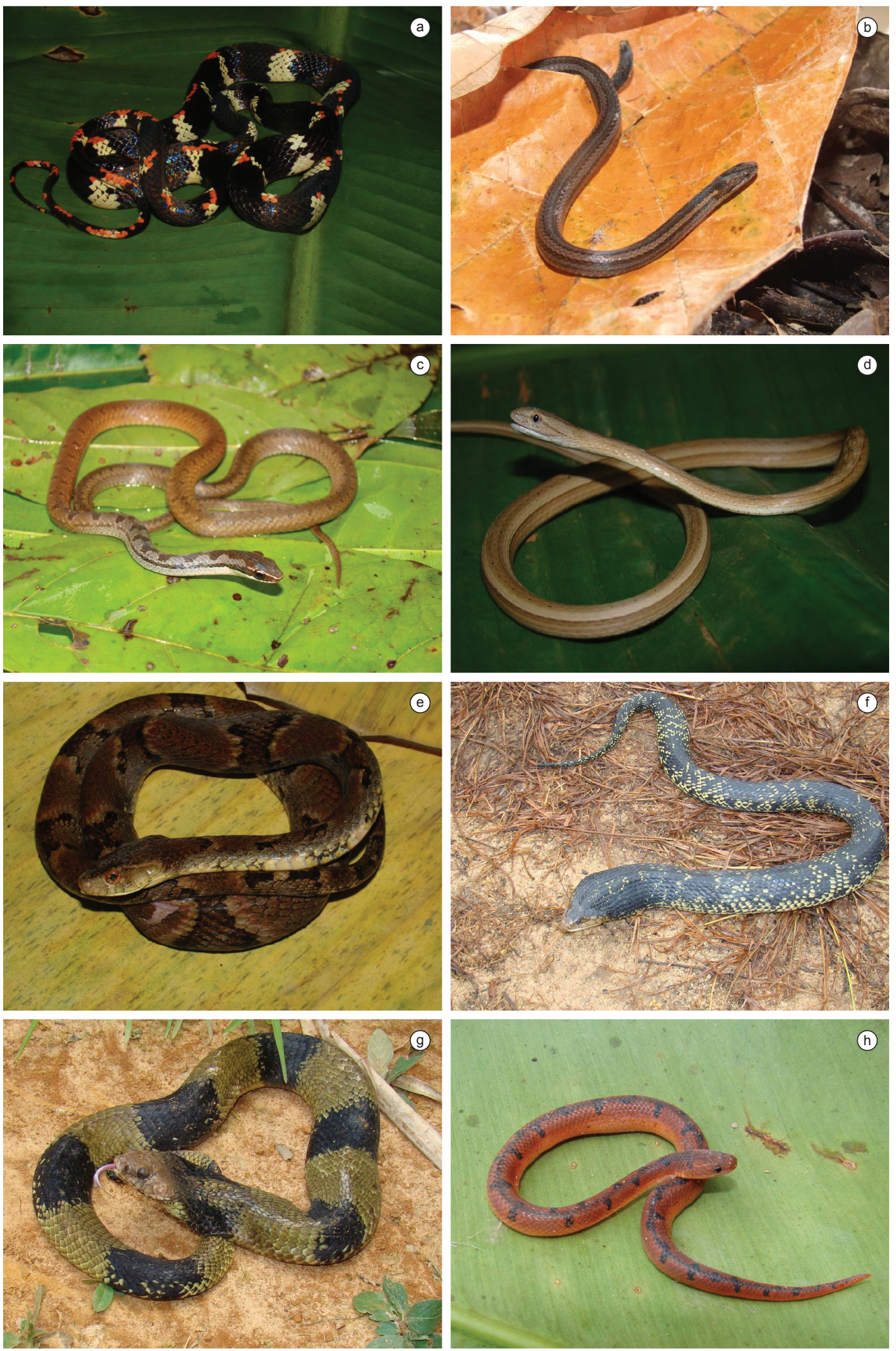

Figura 12. a) Siphlophis worontzowi - Porto Velho (RO). Foto por LT; b) Taeniophalus brevirostris - AM. Foto por PB; c) T. occipitalis - Porto Velho (RO). Foto por TB; d) Thamnodynastes pallidus - Porto Velho (RO); e) Xenodon rabdocephalus - Porto Velho (RO). Fotos por LT; f) e g) X. severus - Porto Velho (RO); h) Xenopholis scalaris - Porto Velho (RO). Fotos por TB.

Figure 12. a) Siphlophis worontzowi - Porto Velho (RO). Photo by LT; b) Taeniophalus brevirostris - AM. Photo by PB; c) T. occipitalis - Porto Velho (RO). Photo by TB; d) Thamnodynastes pallidus - Porto Velho (RO); e) Xenodon rabdocephalus - Porto Velho (RO). Photos by LT; f) e g) X. severus - Porto Velho (RO); h) Xenopholis scalaris - Porto Velho (RO). Photos by TB. 

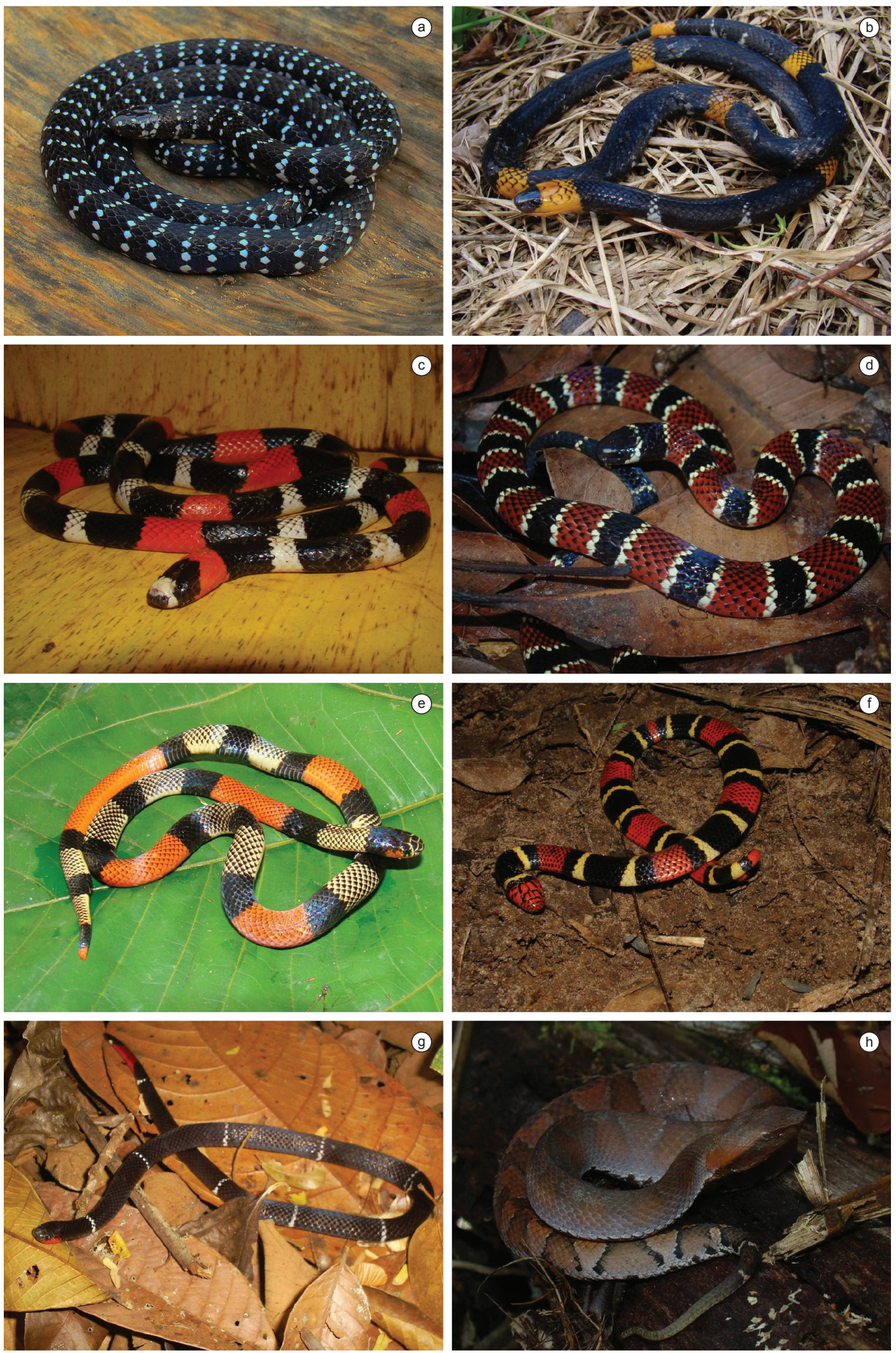

Figura 13. a) Micrurus albicinctus - Porto Velho (RO); b) M. hemprichii - Porto Velho (RO); c) M. lemniscatus - Porto Velho (RO); d) M. cf. ornatissimus - Porto Velho (RO). Fotos por LT; e) M. spixii - Porto Velho (RO). Foto por TB; f) M. surinamensis - Alta Floresta do Oeste (RO); g) Micrurus sp. - Cacoal (RO); h) Bothrocophias hyoprora - Porto Velho (RO). Fotos por LT.

Figure 13. a) Micrurus albicinctus - Porto Velho (RO); b) M. hemprichii - Porto Velho (RO); c) M. lemniscatus - Porto Velho (RO); d) M. cf. ornatissimus - Porto Velho (RO). Photos by LT; e) M. spixii - Porto Velho (RO). Photo by TB; f) M. surinamensis - Alta Floresta do Oeste (RO); g) Micrurus sp. - Cacoal (RO); h) Bothrocophias hyoprora - Porto Velho (RO). Photos by LT. 

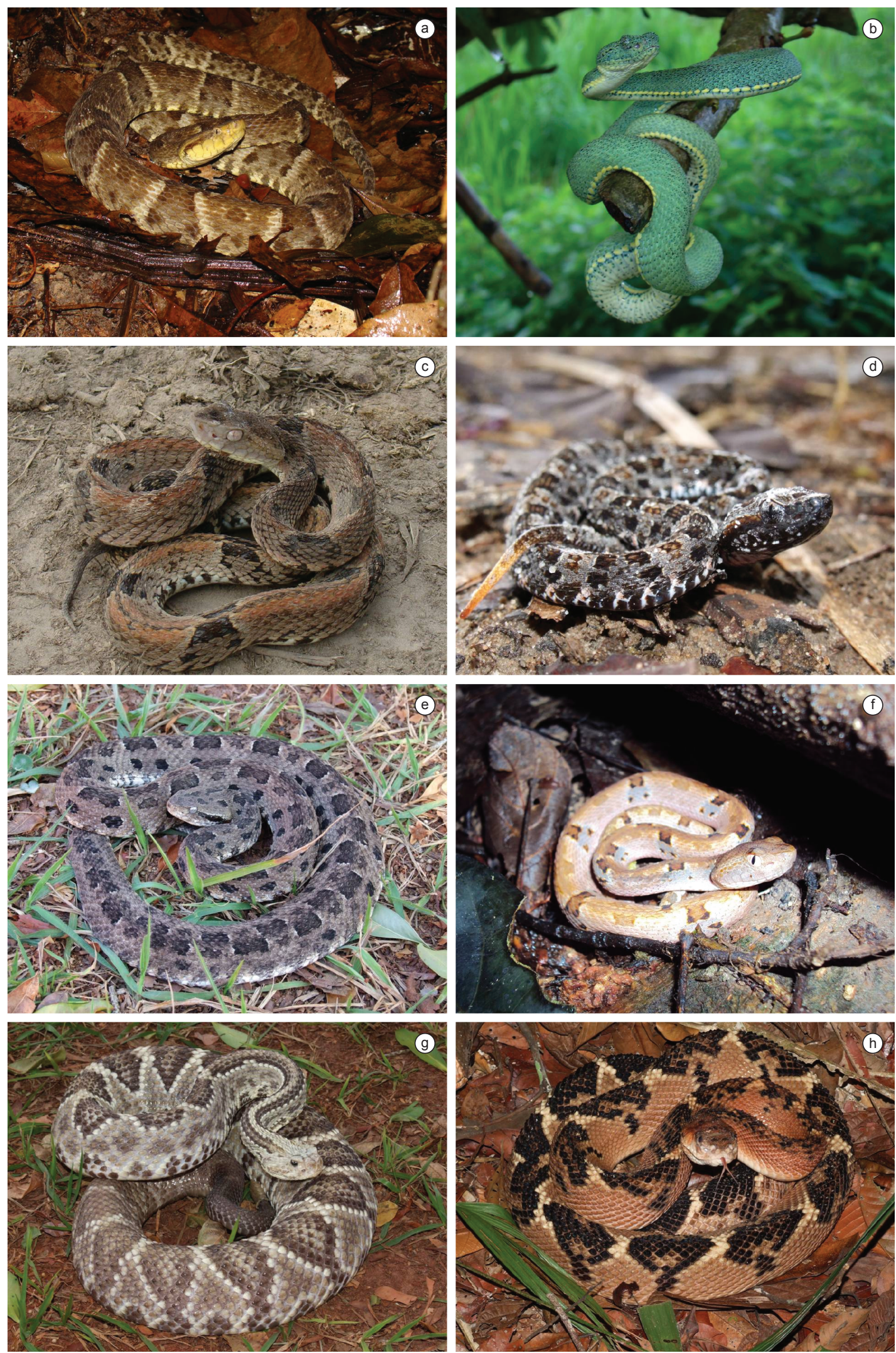

Figura 14. a) Bothrops atrox - Porto Velho (RO). Foto por LT; b) B. bilineata - Porto Velho (RO); c) B. brazili - Porto Velho (RO). Fotos por TB; d) B. mattogrossensis (juvenil) - AM; e) Bothrops mattogrossensis - MS. Fotos por PB; f) B. taeniata - Porto Velho (RO). Foto por TB; g) Crotalus durissus - MS; h) Lachesis muta - AM. Fotos por PB.

Figure 14. a) Bothrops atrox - Porto Velho (RO). Photo by LT; b) B. bilineata - Porto Velho (RO); c) B. brazili - Porto Velho (RO). Photos por TB; d) B. mattogrossensis (juvenile) - AM; e) B. mattogrossensis - MS. Photos by PB; f) B. taeniata - Porto Velho (RO). Photo by TB; g) Crotalus durissus - MS; h) Lachesis muta - AM. Photos by PB. 


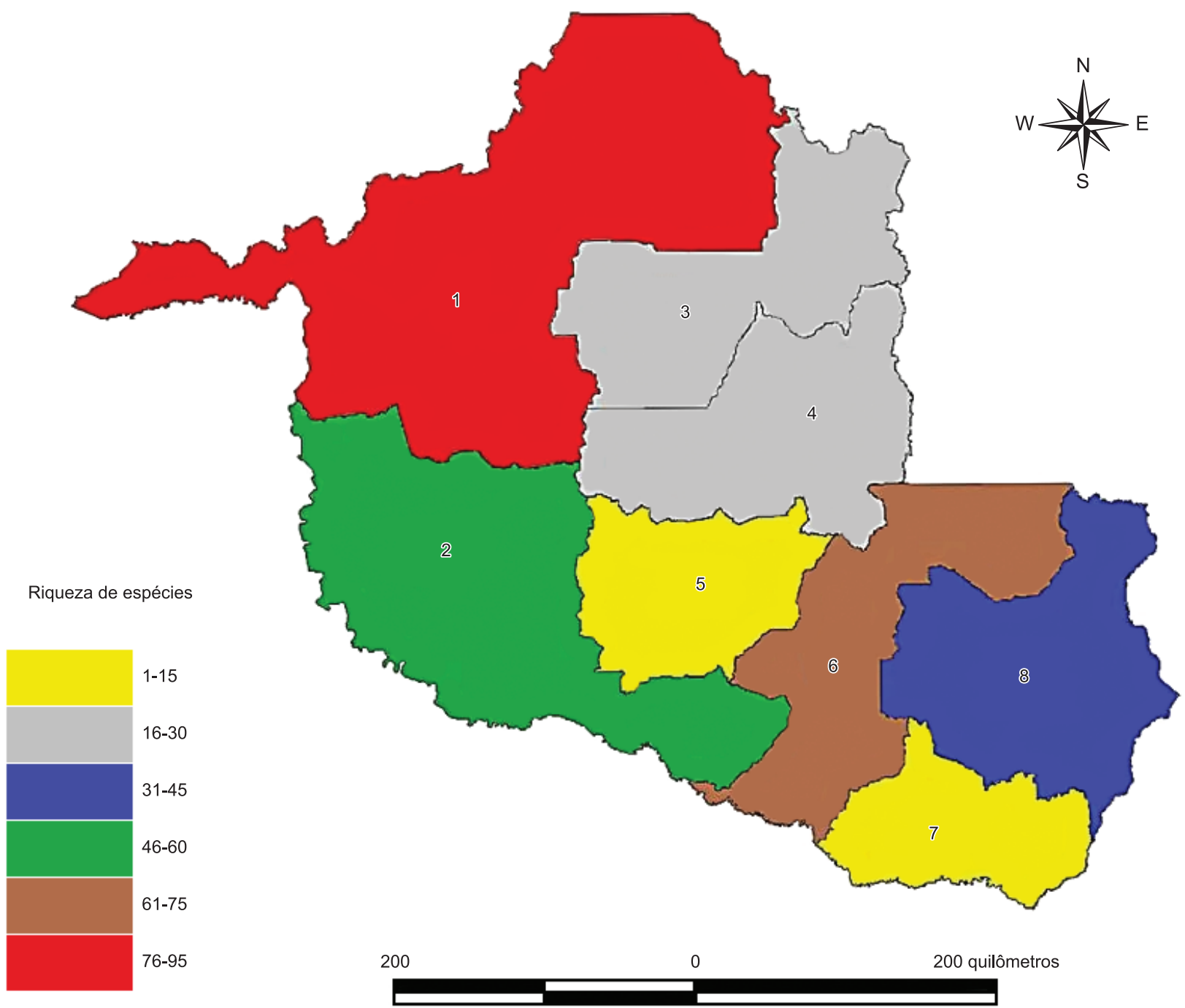

Figura 15. Riqueza de espécies de serpentes registrada nas microrregiões de Rondônia (Brasil). Microrregiões: 1 = Porto Velho; 2 =Guajará-Mirim; 3 =Ariquemes; 4 = Ji-paraná; 5 = Alvorada d'Oeste; $6=$ Cacoal; 7 = Colorado do Oeste; $8=$ Vilhena.

Figure 15. Snakes richness species recorded in the microregions of Rondônia (Brazil). Microregions: $1=$ Porto Velho; $2=$ Guajará-Mirim; $3=$ Ariquemes; 4 = Ji-paraná; $5=$ Alvorada d'Oeste; 6 = Cacoal; 7 = Colorado do Oeste; $8=$ Vilhena.

\section{Lista das Espécies de Serpentes do Estado de Rondônia}

\section{LEPTOTYPHLOPIDAE (4 ESPÉCIES)}

1) Epictia diaplocia (Orejas-Miranda, 1969)

Nome vulgar: Cobra-cega.

Bibliografia: Nascimento et al. (1988) e Jorge-da-Silva (1993).

2) Epictia tenella (Klauber, 1939)

Nome vulgar: Cobra-cega.

Bibliografia: Bernarde \& Abe (2006) e Arredondo \& Zaher (2010).

Comentários: Bernarde \& Abe (2006) registraram essa espécie como Leptotyphlops macrolepis.

3) Siagonodon septemstriatus (Schneider, 1801)

Nome vulgar: Cobra-cega.

Bibliografia: Jorge-da-Silva (1993).
4) Tricheilostoma macrolepis (Peters, 1857)

Nome vulgar: Cobra-cega.

Bibliografia: Vanzolini (1986) e Jorge-da-Silva (1993).

Typhlopidae (1 espécie)

5) Typhlops reticulatus (Linnaeus, 1758)

Nome vulgar: Cobra-cega.

Bibliografia: Dixon \& Hendricks (1979), Nascimento et al. (1988), Jorge-da-Silva (1993), Bernarde \& Abe (2006), França et al. (2006), Turci \& Bernarde (2008) e Marçal et al. (2011).

ANILIIDAE (1)

6) Anilius scytale (Linnaeus, 1758)

Nome vulgar: Coral, cobra-coral e falsa-coral.

Bibliografia: Vanzolini (1986), Jorge-da-Silva (1993), Bernarde $\&$ Abe (2006), Turci \& Bernarde $(2008,2009)$ e Marçal et al. (2011). 


\section{BOIDAE (6 ESPÉCIES)}

7) Boa constrictor (Linnaeus, 1758)

Nome vulgar: Jiboia.

Bibliografia: Vanzolini (1986), Jorge-da-Silva (1993), Brandão (2002), Bernarde \& Abe (2006), França et al. (2006), Turci \& Bernarde (2008, 2009), Bernarde \& Abe (2010) e Marçal et al. (2011).

8) Corallus batesii (Gray, 1860)

Nome vulgar: Bico-de-papagaio, cobra-papagaio e periquitamboia.

Bibliografia: Vanzolini (1986), Jorge-da-Silva (1993), Yuki et al. (1999), Bernarde \& Abe (2006), Turci \& Bernarde (2008), Avila-Pires et al. (2009), Henderson et al. (2009) e Marçal et al. (2011).

9) Corallus hortulanus (Linnaeus, 1758)

Nome vulgar: Suaçuboia.

Bibliografia: Vanzolini (1986), Nascimento et al. (1988), Jorge-da-Silva (1993), Henderson (1997), Yuki et al. (1999), Brandão (2002), Bernarde \& Abe (2006), Avila-Pires et al. (2009), Bernarde \& Abe (2010) e Marçal et al. (2011).

10) Epicrates cenchria (Linnaeus, 1758)

Nome vulgar: Salamanta.

Bibliografia: Vanzolini (1986), Nascimento et al. (1988), Jorgeda-Silva (1993), Bernarde \& Abe (2006), Passos \& Fernandes (2009), Turci \& Bernarde (2008, 2009), Avila-Pires et al. (2009), Bernarde \& Abe (2010) e Marçal et al. (2011).

11) Epicrates crassus (Cope, 1862)

Nome vulgar: Salamanta.

Bibliografia: Jorge-da-Silva (1993) e Passos \& Fernandes (2009).

12) Eunectes murinus (Linnaeus, 1758)

Nome vulgar: Sucuri.

Bibliografia: Amaral (1925, 1948), Jorge-da-Silva (1993), Brandão (2002), Macedo-Bernarde (2006), Bernarde \& Abe (2006; 2010) e Marçal et al. (2011).

\section{COLUBRIDAE (21 ESPÉCIES)}

13) Chironius exoletus (Linnaeus, 1758)

Nome vulgar: Cobra-cipó.

Bibliografia: Vanzolini (1986), Nascimento et al. (1988), Dixon et al. (1993), Jorge-da-Silva (1993), Yuki et al. (1999), Bernarde \& Abe (2006), Turci \& Bernarde (2008), Bernarde \& Abe (2010), Gusmão et al. (2011) e Marçal et al. (2011).

14) Chironius carinatus (Linnaeus, 1758)

Nome vulgar: Cobra-cipó.

Bibliografia: Marçal et al. (2011).

15) Chironius flavolineatus (Boettger, 1885)

Nome vulgar: Cobra-cipó.

Bibliografia: Albuquerque \& Freitas (2011).

16) Chironius fuscus (Linnaeus, 1758)

Nome vulgar: Cobra-cipó.

Bibliografia: Vanzolini (1986), Nascimento et al. (1988), Jorgeda-Silva (1993), Yuki et al. (1999), Avila-Pires et al. (2009) e Marçal et al. (2011).
17) Chironius multiventris (Schmidt \& Walker, 1943)

Nome vulgar: Cobra-cipó.

Bibliografia: Vanzolini (1986), Nascimento et al. (1988), Jorgeda-Silva (1993), Bernarde \& Abe (2006), Avila-Pires et al. (2009) e Marçal et al. (2011).

18) Chironius scurrulus (Wagler, 1824)

Nome vulgar: Cobra-cipó.

Bibliografia: Vanzolini (1986), Nascimento et al. (1988), Jorgeda-Silva (1993), Yuki et al. (1999), Bernarde \& Abe (2006), AvilaPires et al. (2009), Bernarde \& Abe (2010) e Marçal et al. (2011).

19) Dendrophidion dendrophis (Schlegel, 1837)

Nome vulgar: Cobra-cipó.

Bibliografia: Vanzolini (1986), Jorge-da-Silva (1993), Bernarde \& Abe (2006). Avila-Pires et al. (2009) e Bernarde \& Abe (2010).

20) Drymarchon corais (Boie, 1827)

Nome vulgar: Limpa-pasto.

Bibliografia: Vanzolini (1986), Nascimento et al. (1988), Jorgeda-Silva (1993), Bernarde \& Abe (2006), França et al. (2006), Turci \& Bernarde $(2008,2009)$ e Bernarde \& Abe (2010).

21) Drymobius rhombifer (Günther, 1860)

Bibliografia: Vanzolini (1986), Jorge-da-Silva (1993), Bernarde $\&$ Abe (2006).

Comentários: Espécie com poucos registros para o Brasil (Vanzolini 1986, O’Shea \& STIMSON 1993, Bernarde \& Abe 2006, Bernarde et al. 2011a), ocorrendo indivíduos com manchas romboidais ao longo do corpo (Figura 4a) e também com padrão ausente de manchas (O'Shea \& STIMSON 1993, P. S. Bernarde, Obs. pess.).

22) Drymoluber brazili (Gomes, 1918)

Bibliografia: França et al. (2006).

23) Drymoluber dichrous (Peters, 1863)

Bibliografia: Vanzolini (1986), Nascimento et al. (1988), Jorgeda-Silva (1993), Bernarde \& Abe (2006), Avila-Pires et al. (2009), Bernarde \& Abe (2010) e Marçal et al. (2011).

24) Leptophis ahaetulla (Linnaeus, 1758)

Nome vulgar: Cobra-cipó.

Bibliografia: Vanzolini (1986), Nascimento et al. (1988), Jorgeda-Silva (1993), Bernarde \& Abe (2006), Albuquerque et al. (2007), Turci \& Bernarde (2008, 2009) e Marçal et al. (2011).

25) Masticophis mentovarius (Duméril, Bibron \& Duméril, 1854)

Bibliografia: Bernarde \& Abe (2006, 2010).

Comentários: Espécie com poucos registros para o Brasil (Bernarde \& Abe 2006).

26) Mastigodryas boddaerti (Sentzen, 1796)

Nome vulgar: Jararaca.

Bibliografia: Vanzolini (1986), Nascimento et al. (1988), Jorge-da-Silva (1993), Bernarde \& Abe (2006), Turci \& Bernarde (2008), Bernarde \& Abe (2010) e Marçal et al. (2011).

27) Oxybelis aeneus (Wagler, 1824)

Nome vulgar: Cobra-cipó.

Bibliografia: Vanzolini (1986), Jorge-da-Silva (1993), França et al. (2006) e Marçal et al. (2011).

28) Oxybelis fulgidus (Daudin, 1803)

Nome vulgar: Bicuda, cobra-verde e cobra-cipó. 
Bibliografia: Vanzolini (1986), Nascimento et al. (1988), Jorgeda-Silva (1993), Whittaker (1996), Bernarde \& Abe (2006, 2010) e Marçal et al. (2011).

29) Pseustes poecilonotus (Günther, 1858)

Nome vulgar: Papa-ovo e papa-pinto.

Bibliografia: Vanzolini (1986), Jorge-da-Silva (1993), Bernarde \& Abe (2006), Avila-Pires et al. (2009), Bernarde \& Abe (2010) e Marçal et al. (2011).

30) Pseustes sulphureus (Wagler, 1824)

Nome vulgar: Papa-ovo e papa-pinto.

Bibliografia: Jorge-da-Silva (1993) e Marçal et al. (2011).

31) Rhinobothryum lentiginosum (Scopoli, 1785)

Nome vulgar: Coral e falsa-coral

Bibliografia: Jorge-da-Silva (1993), Bernarde \& Abe (2006), França et al. (2006), Turci \& Bernarde (2008), Avila-Pires et al. (2009), Bernarde \& Abe (2010) e Marçal et al. (2011).

32) Spilotes pullatus (Linnaeus, 1758)

Nome vulgar: Caninana.

Bibliografia: Vanzolini (1986), Jorge-da-Silva (1993), Brandão (2002), Bernarde \& Abe (2006), Turci \& Bernarde (2009) e Marçal et al. (2011).

33) Tantilla melanocephala (Linnaeus, 1758)

Bibliografia: Vanzolini (1986), Jorge-da-Silva (1993), Bernarde $\&$ Abe (2006), França et al. (2006), Avila-Pires et al. (2009) e Marçal et al. (2011).

\section{DIPSADIDAE (67 ESPÉCIES)}

34) Apostolepis nigrolineata (Peters, 1869)

Bibliografia: Amaral (1925), Jorge-da-Silva (1993), Lema \& Renner (1998), Lema \& Albuquerque (2010), Curcio et al. (2011) e Marçal et al. (2011).

Comentários: Amaral (1925) descreveu essa espécie como Apostolepis rondoni, sendo considerada sinônima de A. nigrolineata por Lema \& Albuquerque (2010). Lema \& Albuquerque (2010) revalidaram Apostolepis pymi Boulenger, 1903. Posteriormente, Curcio et al. (2011) consideraram A. pymi como sinônima de $A$. nigrolineata.

35) Apostolepis quinquelineata (Boulenger, 1896)

Bibliografia: Jorge-da-Silva (1993), Lema \& Renner (1998), Lema \& Albuquerque (2010) e Curcio et al. (2011).

Comentários: Lema \& Albuquerque (2010) consideraram Apostolepis quinquelineata como um sinônimo júnior de A. pymi, entretanto, Curcio et al. (2011) consideraram A. quinquelineata uma espécie válida e $A$. pymi sinônima de $A$. nigrolineata.

36) Apostolepis striata (Lema, 2004)

Bibliografia: Lema (2004).

Comentários: Essa espécie foi descrita com base em apenas um exemplar e conhecida somente para o cerrado de Vilhena (Lema 2004).

37) Atractus albuquerquei (Cunha \& Nascimento, 1983)

Bibliografia: Vanzolini (1986), Nascimento et al. (1988), Jorge-da-Silva (1993), Jorge-da-Silva et al. (2005), Zaher et al. (2005), Bernarde \& Abe (2006), Turci \& Bernarde (2008) e Marçal et al. (2011).

38) Atractus elaps (Günther, 1858)

Nome vulgar: Coral, cobra-coral e falsa-coral.
Bibliografia: Vanzolini (1986), Jorge-da-Silva (1993), Jorgeda-Silva et al. (2005) e Zaher et al. (2005).

39) Atractus flammigerus (Boie, 1827)

Bibliografia: Jorge-da-Silva (1993).

40) Atractus insipidus (Roze, 1961)

Bibliografia: Jorge-da-Silva (1993) e Jorge-da-Silva et al. (2005).

Comentários: Jorge-da-Silva (1993) registrou essa espécie como Atractus cf. insipidus, uma vez que os 12 exemplares examinados (todos fêmeas) apresentaram 19-24 subcaudais, enquanto o espécime-tipo apresenta 35 . O exemplar fotografado em Porto Velho (Figura 6a) possui 37 subcaudais, demonstrando ser $A$. insipidus.

41) Atractus latifrons (Günther, 1868)

Nome vulgar: Coral, cobra-coral e falsa-coral.

Bibliografia: Vanzolini (1986), Jorge-da-Silva (1993), Bernarde $\&$ Abe (2006), Avila-Pires et al. (2009) e Bernarde \& Abe (2010).

Comentários: Essa serpente é mímica de Micrurus (Campbell \& Lamar 2004) apresentando formas com padrão coralino tendo anéis vermelhos, pretos e brancos ao longo do corpo (Figura 6c) e também um padrão mímico de Micrurus albicinctus com anéis negros separados por anéis brancos curtos (Figura 6b).

42) Atractus major (Boulenger, 1894)

Bibliografia: Avila-Pires et al. (2009).

43) Atractus schach (Boie, 1827)

Bibliografia: Nascimento et al. (1988) e Jorge-da-Silva (1993).

44) Atractus snethlageae (Cunha \& Nascimento, 1983)

Bibliografia: Vanzolini (1986), Jorge-da-Silva (1993), Bernarde \& Abe (2006), Avila-Pires et al. (2009) e Marçal et al. (2011).

45) Atractus taeniatus (Griffin, 1916)

Bibliografia: Jorge-da-Silva (1993) e Jorge-da-Silva et al. (2005).

46) Clelia clelia (Daudin, 1803)

Nome vulgar: Muçurana e cobra-preta.

Bibliografia: Avila-Pires et al. (2009) e Marçal et al. (2011).

47) Clelia plumbea (Wied, 1820)

Nome vulgar: Muçurana e cobra-preta.

Bibliografia: Vanzolini (1986), Jorge-da-Silva (1993), Zaher (1996), Bernarde \& Abe (2006), França et al. (2006) e Bernarde $\&$ Abe (2010).

Comentários: Bernarde \& Abe (2006) registraram essa espécie como Clelia sp.

48) Dipsas catesbyi (Sentzen, 1796)

Nome vulgar: Dormideira e jararaquinha.

Bibliografia: Vanzolini (1986), Nascimento et al. (1988), Jorgeda-Silva (1993), Bernarde \& Abe (2006), França et al. (2006), Turci \& Bernarde (2008, 2009), Lima \& Prudente (2009), AvilaPires et al. (2009), Bernarde \& Abe (2010) e Marçal et al. (2011).

49) Dipsas indica (Laurenti, 1768)

Nome vulgar: Dormideira e jararaquinha.

Bibliografia: Vanzolini (1986), Jorge-da-Silva (1993), Bernarde \& Abe (2006), Avila-Pires et al. (2009), Bernarde \& Abe (2010) e Marçal et al. (2011).

50) Dipsas pavonina (Schlegel, 1837)

Nome vulgar: Dormideira e jararaquinha. 
Bibliografia: Vanzolini (1986), Jorge-da-Silva (1993), Bernarde \& Abe (2006) e Lima \& Prudente (2009).

51) Dipsas variegata (Duméril, Bibron \& Duméril, 1854)

Nome vulgar: Dormideira e jararaquinha.

Bibliografia: Passos et al. (2004), Avila-Pires et al. (2009) e Fernandes et al. (2010).

52) Drepanoides anomalus (Jan, 1863)

Nome vulgar: Coral, cobra-coral e falsa-coral.

Bibliografia: Vanzolini (1986), Jorge-da-Silva (1993), Yuki et al. (1999), Bernarde \& Abe (2006), Turci \& Bernarde (2008), Avila-Pires et al. (2009), Bernarde \& Abe (2010) e Marçal et al. (2011).

Comentários: Em Rondônia são encontrados indivíduos dessa espécie com faixa nucal branca (Figura 7d) e também escura (Figura 7e).

53) Erythrolamprus aesculapii (Linnaeus, 1766)

Nome vulgar: Coral, cobra-coral e falsa-coral.

Bibliografia: Nascimento et al. (1988), Jorge-da-Silva (1993) e Bernarde \& Abe $(2006 ; 2010)$.

54) Erythrolamprus mimus (Cope, 1868)

Nome vulgar: Coral, cobra-coral e falsa-coral.

Bibliografia: Vanzolini (1986), Jorge-da-Silva (1993) e Marçal et al. (2011).

55) Helicops angulatus (Linnaeus, 1758)

Nome vulgar: Cobra-d'água.

Bibliografia: Vanzolini (1986), Jorge-da-Silva (1993), Brandão (2002), Bernarde \& Abe (2006), França et al. (2006), AvilaPires et al. (2009) e Marçal et al. (2011).

56) Helicops hagmanni (Roux, 1910)

Nome vulgar: Cobra-d'água.

Bibliografia: Jorge-da-Silva (1993) e Avila-Pires et al. (2009).

57) Helicops leopardinus (Schlegel, 1837)

Nome vulgar: Cobra-d'água.

Bibliografia: Griffin (1916), Frota (2005) e Marçal et al. (2011).

58) Helicops polylepis (Günther, 1861)

Nome vulgar: Cobra-d'água.

Bibliografia: Griffin (1916) e Jorge-da-Silva (1993).

59) Hydrodynastes bicinctus (Herrmann, 1804)

Nome vulgar: Coral-d'água e cobra-d'água.

Bibliografia: Franco et al. (2007).

60) Hydrodynastes gigas (Duméril, Bibron \& Duméril, 1854)

Bibliografia: Bernarde \& Moura-Leite (1999), Yuki et al. (1999), Brandão (2002), Bernarde \& Abe (2006) e Marçal et al. (2011).

61) Hydrops martii (Wagler, 1824)

Nome vulgar: Coral-d'água e cobra-d'água.

Bibliografia: Brandão (2002) e Marçal et al. (2011).

62) Hydrops triangularis (Wagler, 1824)

Nome vulgar: Coral-d'água e cobra-d'água.

Bibliografia: Vanzolini (1986), Jorge-da-Silva (1993) e Albuquerque \& Lema (2008).

63) Imantodes cenchoa (Linnaeus, 1758)

Nome vulgar: Cobra-cipó.
Bibliografia: Vanzolini (1986), Jorge-da-Silva (1993), Yuki et al. (1999), Bernarde \& Abe (2006), Avila-Pires et al. (2009) e Marçal et al. (2011).

64) Imantodes lentiferus (Cope, 1894)

Nome vulgar: Cobra-cipó.

Bibliografia: Jorge-da-Silva (1993) e Avila-Pires et al. (2009).

65) Leptodeira annulata (Linnaeus, 1758)

Nome vulgar: Jararaca.

Bibliografia: Vanzolini (1986), Jorge-da-Silva (1993), Yuki et al. (1999), Bernarde \& Abe (2006), França et al. (2006), Turci \& Bernarde (2008), Avila-Pires et al. (2009), Bernarde \& Abe (2010) e Marçal et al. (2011).

66) Liophis almadensis (Wagler, 1824)

Nome vulgar: Jararaquinha e cobra-de-capim.

Bibliografia: Jorge-da-Silva (1993), Bernarde \& Abe (2006), França et al. (2006), Turci \& Bernarde (2008) e Bernarde \& Abe (2010).

67) Liophis longiventris (Amaral, 1925)

Nome vulgar: Jararaquinha e cobra-de-capim.

Bibliografia: Vanzolini (1986), Jorge-da-Silva (1993) e França et al. (2006).

68) Liophis miliaris (Linnaeus, 1758)

Nome vulgar: Jararaquinha e cobra-de-capim.

Bibliografia: Vanzolini (1986) e Jorge-da-Silva (1993).

69) Liophis oligolepis (Boulenger, 1905)

Nome vulgar: Jararaquinha e cobra-de-capim.

Bibliografia: Nascimento et al. (1988) e Jorge-da-Silva (1993).

Comentários: Dixon (1983) considerou Liophis oligolepis como sinônimo júnior de $L$. reginae semilineata, entretanto Cunha \& Nascimento (1993) e Jorge-da-Silva (1993) consideraram essa espécie válida baseados em evidentes diferenças na folidose entre elas.

70) Liophis poecilogyrus (Wied, 1825)

Nome vulgar: Jararaquinha e cobra-de-capim.

Bibliografia: Jorge-da-Silva (1993) e Marçal et al. (2011).

71) Liophis reginae (Linnaeus, 1758)

Nome vulgar: Jararaquinha e cobra-de-capim.

Bibliografia: Vanzolini (1986), Nascimento et al. (1988), Jorge-da-Silva (1993), Bernarde \& Abe (2006), Turci \& Bernarde (2008, 2009), Avila-Pires et al. (2009), Bernarde \& Abe (2010) e Marçal et al. (2011).

72) Liophis typhlus (Linnaeus, 1758)

Nome vulgar: Jararaquinha e cobra-de-capim.

Bibliografia: Vanzolini (1986), Jorge-da-Silva (1993), AvilaPires et al. (2009) e Marçal et al. (2011).

Comentários: Essa espécie apresenta três padrões de colorido (Martins \& Oliveira 1998), tendo indivíduos com coloração verde (Figura 9d), marrom-avermelhado (Figura 9e) e cinza-azulado.

73) Liophis taeniogaster (Jan, 1863)

Nome vulgar: Jararaquinha e cobra-de-capim.

Bibliografia: Fernandes et al. (2002) e Bernarde \& Abe (2006).

Comentários: Bernarde \& Abe (2006) registraram essa espécie como Liophis breviceps.

74) Ninia hudsoni Parker, 1940

Bibliografia: Jorge-da-Silva (1993) e Bernarde \& Abe (2006). 
Comentários: Espécie com poucos registros para o Brasil (Jorge-da-Silva 1993, Bernarde \& Abe 2006).

75) Oxyrhopus formosus (Wied, 1820)

Nome vulgar: Coral, cobra-coral e falsa-coral.

Bibliografia: Avila-Pires et al. (2009).

Comentários: Oxyrhopus formosus apresenta indivíduos adultos com faixas vermelhas e pretas (Figura 9f), diferenciandose de Oxyrhopus occipitalis que é caracterizada pelas mudanças ontogenéticas no padrão de colorido (Hoge et al. 1973, Jorgeda-Silva 1993, Lynch 2009).

76) Oxyrhopus melanogenys (Tschudi, 1845)

Nome vulgar: Coral, cobra-coral e falsa-coral.

Bibliografia: Vanzolini (1986), Nascimento et al. (1988), Jorgeda-Silva (1993), Brandão (2002), Bernarde \& Abe (2006), Turci \& Bernarde (2008, 2009), Bernarde \& Abe (2010) e Marçal et al. (2011).

Comentários: Lynch (2009) mencionou que os exemplares de Oxyrhopus melanogenys melanogenys (Tschudi 1845) apresentados no trabalho de Jorge-da-Silva (1993) tratam-se de $O$. vanidicus. Entretanto, O. vanidicus é caracterizada por apresentar tríades negras ao longo do corpo (Lynch 2009) e os indivíduos coletados na Usina de Samuel apresentam o dorso uniformemente vermelho (Jorge-da-Silva 1993, ver Figura 12 do artigo dessa referência), assim como o indivíduo da Figura 9g.

77) Oxyrhopus occipitalis (Wagler in Spix, 1824)

Nome vulgar: Coral, cobra-coral e falsa-coral.

Bibliografia: Jorge-da-Silva (1993).

78) Oxyrhopus petolarius (Linnaeus, 1758)

Nome vulgar: Coral, cobra-coral e falsa-coral.

Bibliografia: Vanzolini (1986), Jorge-da-Silva (1993), Bernarde $\&$ Abe (2006), Avila-Pires et al. (2009) e Marçal et al. (2011).

79) Oxyrhopus rhombifer (Duméril, Bibron \& Duméril, 1854)

Nome vulgar: Coral, cobra-coral e falsa-coral.

Bibliografia: Vanzolini (1986) e Jorge-da-Silva (1993).

80) Oxyrhopus vanidicus (Lynch, 2009)

Nome vulgar: Coral, cobra-coral e falsa-coral.

Bibliografia: Bernarde \& Abe (2006),

Comentários: Oxyrhopus vanidicus distingue-se das outras espécies por apresentar um padrão colorido de tríades e pela falta de um par de curtas faixas escuras na nuca (Lynch 2009). Um exemplar dessa espécie foi registrado como Oxyrhopus melanogenys por Bernarde \& Abe (2006). Vanzolini (1986) registrou $O$. trigeminus (outra espécie de Oxyrhopus que apresenta tríades) para Rondônia, podendo tratar-se também de $O$. vanidicus que já foi confundida previamente com essa espécie (Lynch 2009).

81) Phalotris nasutus (Gomes, 1915)

Nome vulgar: Coral, cobra-coral e falsa-coral.

Bibliografia: Lema et al. (2005).

82) Philodryas argentea (Daudin, 1803)

Nome vulgar: Cobra-cipó.

Bibliografia: Vanzolini (1986), Nascimento et al. (1988), Jorgeda-Silva (1993), Machado (1993), Yuki et al. (1999), Bernarde \& Abe (2006), Prudente et al. (2008), Avila-Pires et al. (2009), Bernarde \& Abe (2010) e Marçal et al. (2011).

Comentários: Uma das características que distinguem Philodryas argentea de $P$. boulengeri é que a primeira apresenta três faixas verdes no ventre (uma mediana mais fina entre duas mais largas) (Figura 10f) e a última uma única e larga faixa verde (Figura 10h).

83) Philodryas boulengeri (Procter, 1923)

Nome vulgar: Cobra-cipó.

Bibliografia: Keiser (1989), Prudente et al. (2008) e Marçal et al. (2011).

84) Philodryas olfersii (Lichtenstein, 1823)

Nome vulgar: Cobra-verde e cobra-cipó.

Bibliografia: Vanzolini (1986), Jorge-da-Silva (1993), Brandão (2002), Bernarde \& Abe (2006), França et al. (2006), Turci \& Bernarde (2008, 2009), Bernarde \& Abe (2010) e Marçal et al. (2011).

85) Philodryas viridissima (Linnaeus, 1758)

Nome vulgar: Cobra-verde e cobra-cipó.

Bibliografia: Vanzolini (1986), Jorge-da-Silva (1993), Avila-

Pires et al. (2009) e Marçal et al. (2011).

86) Pseudoboa coronata (Schneider, 1801)

Nome vulgar: Coral, cobra-coral e falsa-coral.

Bibliografia: Jorge-da-Silva (1993) e Marçal et al. (2011).

87) Pseudoboa martinsi (Zaher, Oliveira \& Franco, 2008)

Nome vulgar: Coral, cobra-coral e falsa-coral.

Bibliografia: Zaher et al. (2008).

88) Pseudoboa nigra (Duméril, Bibron \& Duméril, 1854)

Nome vulgar: Cobra-preta.

Bibliografia: França et al. (2006).

89) Pseudoeryx plicatilis (Linnaeus, 1758)

Nome vulgar: Cobra-d'água.

Bibliografia: Hoge \& Nina (1964), Scartozzoni et al. (2010)

e Marçal et al. (2011).

Comentários: Além dos registros apresentados por Scartozzoni et al. (2010), existe um exemplar no Museu de História Natural Capão da Imbuia em Curitiba (MHNCI 10445) procedente da Usina Hidrelétrica de Samuel.

90) Siphlophis cervinus (Laurenti, 1768)

Bibliografia: Jorge-da-Silva (1993), Prudente et al. (1998), Avila-Pires et al. (2009) e Marçal et al. (2011).

91) Siphlophis compressus (Daudin, 1803)

Nome vulgar: Coral, cobra-coral e falsa-coral.

Bibliografia: Vanzolini (1986), Jorge-da-Silva (1993), Yuki et al. (1999), Bernarde \& Abe (2006), Avila-Pires et al. (2009) e Marçal et al. (2011).

92) Siphlophis worontzowi (Prado, 1940)

Nome vulgar: Coral, cobra-coral e falsa-coral.

Bibliografia: Jorge-da-Silva (1993), Prudente et al. (1998) e Bernarde \& Abe $(2006 ; 2010)$.

93) Taeniophalus brevirostris (Peters, 1863)

Nome vulgar: Jararaquinha.

Bibliografia: Jorge-da-Silva (1993).

94) Taeniophalus occipitalis (Jan, 1863)

Nome vulgar: Jararaquinha.

Bibliografia: Jorge-da-Silva (1993), Bernarde \& Abe (2006),

França et al. (2006) e Santos-Junior et al. (2008). 
95) Thamnodynastes lanei (Bailey, Thomas \& Silva-Jr, 2005)

Nome vulgar: Jararaquinha.

Bibliografia: Bailey et al. (2005).

96) Thamnodynastes pallidus (Linnaeus, 1758)

Nome vulgar: Jararaquinha.

Bibliografia: Jorge-da-Silva (1993), Yuki et al. (1999) e Marçal et al. (2011).

97) Xenodon merremii (Wagler, 1824)

Nome vulgar: Boipeva.

Bibliografia: Moura-Leite \& Bernarde (1999).

98) Xenodon rabdocephalus (Wied, 1824)

Nome vulgar: Jararaca.

Bibliografia: Vanzolini (1986), Jorge-da-Silva (1993), Bernarde

\& Abe (2006), França et al. (2006) e Marçal et al. (2011).

99) Xenodon severus (Linnaeus, 1758)

Nome vulgar: Jararaca.

Bibliografia: Nascimento et al. (1988), Jorge-da-Silva (1993),

Bernarde \& Abe (2006) e Marçal et al. (2011).

100) Xenopholis scalaris (Wucherer, 1861)

Bibliografia: Vanzolini (1986), Jorge-da-Silva (1993), Bernarde \& Abe (2006), Avila-Pires et al. (2009), Bernarde \& Abe (2010) e Marçal et al. (2011).

\section{ELAPIDAE (9 ESPÉCIES)}

\section{1) Micrurus albicinctus (Amaral, 1925)}

Nome vulgar: Coral, cobra-coral e coral-verdadeira.

Bibliografia: Roze \& Jorge-da-Silva (1990), Jorge-da-Silva

(1993), Avila-Pires et al. (2009) e Marçal et al. (2011).

102) Micrurus hemprichii (Jan, 1858)

Nome vulgar: Coral, cobra-coral e coral-verdadeira.

Bibliografia: Roze \& Jorge-da-Silva (1990), Jorge-da-Silva (1993), Roze (1994, 1996) e Marçal et al. (2011).

Comentários: Roze \& Jorge-da-Silva (1990) descreveram Micrurus rondonianus, que foi invalidada e considerada sinônima de $M$. hemprichii ortoni por Jorge-da-Silva (1993). Posteriormente, Micrurus rondonianus foi considerada uma subespécie de M. hemprichii, M. h. rondonianus (Roze 1994, 1996, Campbell \& Lamar 2004).

103) Micrurus lemniscatus (Linnaeus, 1758)

Nome vulgar: Coral, cobra-coral e coral-verdadeira.

Bibliografia: Vanzolini (1986), Roze \& Jorge-da-Silva (1990), Jorge-da-Silva (1993), Turci \& Bernarde (2008), Avila-Pires et al. (2009) e Marçal et al. (2011).

104) Micrurus mipartitus (Duméril, Bibron \& Duméril, 1854)

Nome vulgar: Coral, cobra-coral e coral-verdadeira.

Bibliografia: Vanzolini (1986) e Jorge-da-Silva (1993).

Comentários: Vanzolini (1986) registrou essa espécie para o Brasil no Rio Candeias em Rondônia. Jorge-da-Silva (1993) e Campbell \& Lamar (2004) examinaram esse exemplar (MZUSP 5930) e confirmaram a identificação dessa espécie.

105) Micrurus paraensis (Cunha \& Nascimento, 1973)

Nome vulgar: Coral, cobra-coral e coral-verdadeira.

Bibliografia: Nascimento et al. (1988), Roze \& Jorge-da-Silva (1990), Jorge-da-Silva (1993), Feitosa et al. (2007) e Marçal et al. (2011)
106) Micrurus cf. ornatissimus (Jan, 1858)

Nome vulgar: Coral, cobra-coral e coral-verdadeira.

Bibliografia: Marçal et al. (2011).

107) Micrurus spixii (Wagler, 1824)

Nome vulgar: Coral, cobra-coral e coral-verdadeira.

Bibliografia: Vanzolini (1986), Nascimento et al. (1988), Roze \& Jorge-da-Silva (1990), Jorge-da-Silva (1993), Bernarde \& Abe (2006), Turci \& Bernarde (2008, 2009), Bernarde \& Abe (2010) e Marçal et al. (2011).

108) Micrurus surinamensis (Cuvier, 1817)

Nome vulgar: Coral, coral-d'água, cobra-coral e coralverdadeira.

Bibliografia: Vanzolini (1986), Roze \& Jorge-da-Silva (1990), Jorge-da-Silva (1993), Passos \& Fernandes (2005), Bernarde \& Abe (2006), Turci \& Bernarde (2008), Avila-Pires et al. (2009) e Marçal et al. (2011).

109) Micrurus sp.

Nome vulgar: Coral, cobra-coral e coral-verdadeira.

Bibliografia: Registrada em Espigão do Oeste e Cacoal como Micrurus hemprichii por Bernarde \& Abe (2006) e Turci \& Bernarde (2008), respectivamente.

Comentários: Trata-se de uma espécie em fase de descrição.

\section{VIPERIDAE (9 ESPÉCIES)}

110) Bothrocophias hyoprora (Amaral, 1935)

Nome vulgar: Jararaca-nariguda e jararaca-bicuda.

Bibliografia: Jorge-da-Silva (1993) e Marçal et al. (2011).

111) Bothrocophias microphthalmus (Cope, 1876)

Bibliografia: Bernarde et al. (2008) e Bernarde et al. (2012).

Comentários: Bernarde et al. (2012) analisaram o exemplar identificado como Bothrocophias hyoprora por Bernarde et al. (2008) para a Reserva Biológica do Jaru (RO) e constataram que o mesmo trata-se de B. microphtalmus. Essa espécie foi assinalada para o Brasil por Hoge \& Romano-Hoge (1981), sem apresentar a localidade de registro. O exemplar UFACF 216 apresenta as seguintes características: supralabiais $8 / 8$; infralabiais $11 / 11$; dorsais $23 / 25 / 21$; ventrais 175 ; anal inteira; subcaudais 48 (maioria dividida); comprimento rostro-anal $30 \mathrm{~cm}$; comprimento da cauda $3,7 \mathrm{~cm}$.

112) Bothrops atrox (Linnaeus, 1758)

Nome vulgar: Jararaca, jararaquinha-do-rabo-branco (juvenil), jararacão.

Bibliografia: Vanzolini (1986), Nascimento et al. (1988), Jorgeda-Silva (1993), Yuki et al. (1999), Macedo-Bernarde \& Bernarde (2005), Bernarde \& Abe (2006), Nascimento et al. (2008), AvilaPires et al. (2009), Bernarde \& Abe (2010) e Marçal et al. (2011).

113) Bothrops bilineata (Wied, 1821)

Nome vulgar: Bico-de-papagaio e cobra-papagaio.

Bibliografia: Jorge-da-Silva (1993), Bernarde \& Abe (2006), Turci \& Bernarde (2008), Avila-Pires et al. (2009), Bernarde et al. (2011b) e Marçal et al. (2011).

Comentários: Duas subespécies de Bothrops bilineata ocorrem em Rondônia (Bernarde et al. 2011), B. b. bilineata que ocorre no centro para o sul de Rondônia e $B$. $b$. smaragdina que ocorre no norte do estado.

114) Bothrops brazili (Hoge, 1954)

Nome vulgar: Jararaca. 
Bibliografia: Vanzolini (1986), Jorge-da-Silva (1993) e Marçal et al. (2011).

115) Bothrops mattogrossensis (Amaral, 1925)

Nome vulgar: Jararaca-pintada.

Bibliografia: Vanzolini (1986), Jorge-da-Silva (1993), França et al. (2006) e Xavier-da-Silva \& Rodrigues (2008).

116) Bothrops taeniata (Wagler, 1824)

Nome vulgar: Jararaca-cinzenta.

Bibliografia: Jorge-da-Silva (1993) e Avila-Pires et al. (2009).

117) Crotalus durissus (Linnaeus, 1758)

Nome vulgar: Cascavel.

Bibliografia: Jorge-da-Silva (1993) e França et al. (2006).

118) Lachesis muta (Linnaeus, 1766)

Nome vulgar: Bico-de-jaca, surucucu-pico-de-jaca e surucucu. Bibliografia: Vanzolini (1986), Jorge-da-Silva (1993), Fernandes et al. (2004) e Bernarde \& Abe (2006, 2010).

\section{Discussão}

A última lista de serpentes para o estado de Rondônia continha 95 espécies (Jorge-da-Silva 1993), sendo acrescentadas 23 neste trabalho, totalizando 118 espécies de serpentes. A riqueza de serpentes em Rondônia corresponde a $31 \%$ da riqueza conhecida para o Brasil (Bérnils \& Costa 2011).

A menor riqueza encontrada em áreas de cerrado (27 espécies) provavelmente deve estar associada aos poucos trabalhos desenvolvidos nessas áreas e pelo fato dessa formação vegetal ocupar cerca de apenas 5\% da área do estado (Oliveira 2002). Entretanto, essa riqueza pode estar subestimada devido à falta de estudos nestas regiões, uma vez que 158 espécies de serpentes são conhecidas em áreas de cerrado no Brasil (Nogueira et al. 2011). Nove espécies (Epicrates crassus, Chironius flavolineatus, Drymoluber brazili, Apostolepis striata, Oxyrhopus rhombifer, Pseudoboa nigra, Xenodon merremii, Bothrops mattogrossensis e Crotalus durissus) foram registradas exclusivamente em áreas de cerrado, sendo formas associadas a esse ambiente na Amazônia (Jorge-da-Silva 1993, Lema 2004, França et al. 2006, Passos \& Fernandes 2009, Xavier-da-Silva \& Rodrigues 2008). Destas, Apostolepis striata é uma forma aparentemente endêmica da porção norte do platô dos Parecis, que abriga outras espécies de Squamata com distribuição restrita (Nogueira et al. 2011). Liophis almadensis é uma espécie associada ao cerrado (França et al. 2006), sendo registrada também em áreas de floresta transformadas em pastagem (Bernarde \& Abe 2006). Philodryas olfersii, na Amazônia, é outra espécie tida como restrita aos enclaves de cerrado (França et al. 2006). Entretanto foi registrada em área de floresta por Bernarde \& Abe (2006). Seis espécies (Masticophis mentovarius, Apostolepis striata, Erythrolamprus mimus, Micrurus mipartitus, Micrurus sp. e Bothrocophias microphthalmus) são conhecidas no Brasil apenas para Rondônia (Vanzolini 1986, Jorge-da-Silva 1993, Lema 2004, Bernarde \& Abe 2006, Bernarde et al. 2012).

As espécies com maior número de indivíduos registrados nos estudos são também as consideradas mais abundantes em outras regiões na Amazônia (Cunha \& Nascimento 1993, Martins \& Oliveira 1998, Frota 2004, Frota et al. 2005, Silva et al. 2010) e em Rondônia são as mais encontradas (com exceção de Xenopholis scalaris) em áreas de pastagens e de vegetação secundária (Jorge-da-Silva 1993, Bernarde \& Abe 2006, Turci \& Bernarde 2008, 2009). Cinquenta e duas espécies foram consideradas pouco frequentes (menos de
5 indivíduos registrados) podendo ser um indicativo da necessidade de levantamentos em Rondônia.

As microrregiões melhores amostradas em Rondônia são a de Porto Velho, Guajará-Mirim e de Cacoal, onde se concentram a maior parte dos inventários desenvolvidos (e. g., Jorge-da-Silva 1993, Bernarde \& Abe 2006, Avila-Pires et al. 2009). Alvorada d'Oeste, Colorado do Oeste e Ariquemes são microrregiões com pouca amostragem, onde devem ser concentrados esforços de coleta no futuro para preencher essas lacunas de conhecimento. Apesar da microrregião de Guajará-Mirim ter sido a terceira melhor amostrada (55 espécies), o principal levantamento foi realizado na Bacia do Mamoré (Avila-Pires et al. 2009), permanecendo a Bacia do Guaporé subamostrada. As microrregiões de Colorado do Oeste e de Vilhena, situadas no sul do estado com principal concentração de áreas de Cerrado (Oliveira 2002), também se encontram subamostradas. É importante que estudos herpetofaunísticos sejam dirigidos para essas áreas uma vez que o avanço da fronteira agrícola no Cerrado e a consequente perda de habitats é a principal ameaça para herpetofauna nesta região (Gainsbury \& Colli 2003, Nogueira et al. 2010, 2011).

Em Rondônia são necessários mais estudos herpetofaunísticos, sendo um dos indicadores disso a presença de alguns táxons pouco colecionados, que representam ocorrências importantes para o Brasil (e. g., Apostolepis striata, Erythrolamprus mimus, Ninia hudsoni, Drymobius rhombifer, Masticophis mentovarius. Micrurus mipartitus e Bothrocophias microphthalmus) e que requerem maiores informações taxonômicas, ecológicas e zoogeográficas. Associa-se essa necessidade de mais estudos com o fato de algumas regiões não terem sido amostradas, um quadro que se agrava, uma vez que Rondônia tem sua biodiversidade ameaçada nas manchas de cerrado pelo avanço da agricultura mecanizada (e. g., Colli et al. 2003, Gainsbury \& Colli 2003, França et al. 2006) e diminuição da riqueza nas áreas onde a cobertura florestal foi transformada em pastagens (Bernarde 2007, Bernarde \& Macedo 2008, Macedo et al. 2008).

\section{Agradecimentos}

Ao Nelson Rufino de Albuquerque, Márcia Renner e Thales de Lema pelo envio de bibliografias e informações sobre as espécies de Apostolepis. Ao CNPq pela bolsa de produtividade em pesquisa para PSB (501927/2009-3). Aos revisores anônimos pelas valiosas sugestões.

\section{Referências Bibliográficas}

ADALSTEINSSON, S.A., BRANCH, W.R., TRAPÉ, S., VITT, L.J. \& HEDGES, S.B. 2009. Molecular phylogeny, classification, and biogeography of snakes of the Family Leptotyphlopidae (Reptilia, Squamata). Zootaxa 2244:1-50.

ALBUQUERQUE, N.R. \& LEMA, T. 2008. Taxonomic revision of the Neotropical water snake Hydrops triangularis. Zootaxa 1685:55-66.

ALBUQUERQUE, N.R., GALATTI, U. \& DI-BERNARDO, M. 2007. Diet and feeding behaviour of the Neotropical parrot snake (Leptophis ahaetulla) in northern Brazil. J. Nat. Hist. 41(17-20):1237-1243.

ALBUQUERQUE, S. \& FREITAS, M.A. 2011. Chironius flavolineatus (Liana snake): distribution extension in Rondônia State. Herpetol. Rev. 42(4):570-571.

AMARAL, A. 1925. Ophidios de Matto Grosso (Contribuição II para o conhecimento dos ophidios do Brasil). Comm. Linhas Telegraph. Estrat. Matto Grosso ao Amazonas. Hist. Natural. Zool. 84(5):1-29.

AMARAL, A. 1948. Ofídios de Mato Grosso. 2. ed. Comissão Linhas Telegráficas Estratégicas do Mato Grosso e Amazonas, Imprensa Nacional, Rio de Janeiro. Publicação n. 84, Anexos n. 5, Hist. Nat. Zool.

ARREDONDO, J.C. \& ZAHER, H. 2010. A new species of Epictia (Serpentes: Leptotyphlopidae) from Central Brazil. South American. J. Herpetol. 5(3):189-198. http://dx.doi.org/10.2994/057.005.0304 
AVILA-PIRES, T.C.S., HOOGMOED, M.S. \& VITT, L.J. 2007. In Herpetofauna da Amazônia. Herpetologia no Brasil II (L.B. Nascimento \& M.E. Oliveira, eds.). Sociedade Brasileira de Herpetologia, Belo Horizonte, p.13-43.

AVILA-PIRES, T.C.S., VITT, L.J., SARTORIUS, S.S. \& ZANI, P.A. 2009. Squamata (Reptilia) from four sites in southern Amazonia, with a biogeographic analysis of Amazonian lizards. Bol. Mus. Para. Emílio Goeldi Ciênc. Nat. 4(2):99-118.

AVILA-PIRES, T.C.S., HOOGMOED, M.S. \& ROCHA, W.A. 2010. Notes on the vertebrates of northern Pará, Brazil: a forgotten part of the Guianan Region, I. Herpetofauna. Bol. Mus. Para. Emílio Goeldi Ciênc. Nat. 5:13-112.

BAILEY, J.R., THOMAS, R.A. \& JORGE-DA-SILVA JUNIOR, N. 2005. A revision of the South American snake genus Thamnodynastes Wagler, 1830 (Serpentes, Colubridae, Tachymenini). I. Two new species of Thamnodynastes from Central Brazil and adjacente areas, with a redefinition of and neotype designation for Thamnodynastes pallidus (Linnaeus, 1758). Phyllomedusa. 4(2):83-101.

BERNARDE, P.S. 2007. Ambientes e temporada de vocalização da anurofauna no Município de Espigão do Oeste, Rondônia, Sudoeste da Amazônia - Brasil (Amphibia: Anura). Biota Neotrop. 7(2): http://www. biotaneotropica.org.br/v7n2/pt/abstract?article+bn01507022007 (último acesso em 20/04/2011).

BERNARDE, P.S. \& ABE, A.S. 2006. A snake community at Espigão do Oeste, Rondônia, Southwestern Amazon, Brazil. South Am. J. Herpetol. 1(2):102-113.

BERNARDE, P.S. \& ABE, A.S. 2010. Hábitos alimentares de serpentes em Espigão do Oeste, Rondônia, Brasil. Biota Neotrop. 10(1): http://www. biotaneotropica.org.br/v10n1/pt/abstract?article+bn02510012010 (último acesso em 20/04/2011).

BERNARDE, P.S. \& MACEDO, L.C. 2008. Impacto do desmatamento e formação de pastagens sobre a anurofauna de serapilheira em Rondônia. Iheringia. 98(4):454-459. http://dx.doi.org/10.1590/S007347212008000400006

BERNARDE, P.S. \& MOURA-LEITE, J.C. 1999. Geographic distribution: Hydrodynastes gygas (Surucucu-do-pantanal). Herpetol. Rev. 30:54.

BERNARDE, P.S., FERREIRA-MARTINS, L.S. \& OLIVEIRA, J.R. 2008. Bothrocophias hyoprora (Amazonian Hog-nosed Pit Viper). Diet. Herpetol. Rev. 39(3):353.

BERNARDE, P.S., MACHADO, R.A. \& TURCI, L.C.B. $2011 \mathrm{a}$. Herpetofauna da área do Igarapé Esperança na Reserva Extrativista Riozinho da Liberdade, Acre - Brasil. Biota Neotrop. 11(3): http://www. biotaneotropica.org.br/v11n3/pt/abstract?article+bn02111032011 (último acesso em 20/05/2012)

BERNARDE, P.S., COSTA, H.C., MACHADO, R.A. \& SÃO PEDRO, V.A. 2011b. Bothriopsis bilineata bilineata (Wied, 1821) (Serpentes: Viperidae): New records in the states of Amazonas, Mato Grosso and Rondônia, northern Brazil. CheckList 7(3):343-347.

BERNARDE, P.S., ALBUQUERQUE, S. \& TURCI, L.C.B. 2012. Serpentes peçonhentas e acidentes ofídicos em Rondônia. Anolis Books, Curitiba, 128p.

BÉRNILS, R.S. \& COSTA, H.C. 2011. Brazilian reptiles - List of species. Sociedade Brasileira de Herpetologia. http://www.sbherpetologia.org.br/ (último acesso em 10/06/2012).

BRANDÃO, R.A. 2002. Avaliação ecológica rápida da herpetofauna nas reservas extrativistas de Pedras Negras e Curralinho, Costa Marques, RO. Brasil Florestal. 74:61-73.

CAMPBELL, J.A. \& LAMAR, W.W. 2004. The venomous reptiles of Latin América. Cornell Univ. Press, Ithaca, 425p.

CARRASCO, P.A., MATTONI, C.I., LEYNAUD, G.C. \& SCROCCHI, G.J. 2012. Morphology, phylogeny and taxonomy of South American bothropoid pitvipers (Serpentes, Viperidae). Zool. Scr. 41(2):109-124.
COLLI, G.R., COSTA, G.C., GARDA, A.A., KOPP, K.A., MESQUITA, D.O., PÉRES-JUNIOR, A.K., VALDUJO, P.H., VIEIRA, G.H.C. \& WIEDERHECKER, H.C. 2003. A critically endangered new species of Cnemidophorus (Squamata, Teiidae) from a Cerrado enclave in southwestern Amazonia, Brazil. Herpetologica 59(1):76-88.

CUNHA, O.R. \& NASCIMENTO, F.P. 1978. Ofídios da Amazônia. X - As cobras da Região Leste do Pará. Publ. Avul. Mus. Par. Emílio Goeldi 31:1218.

CUNHA, O.R. \& NASCIMENTO, F.P. 1993. Ofídios da Amazônia: as cobras da região Leste do Pará. Bol. Mus. Para. Emílio Goeldi, Sér. Zool. 9(1):1-191.

CURCIO, F.F., NUNES, P.M.S., HARVEY, M.B. \& RODRIGUES, M.T. 2011. Redescription of Apostolepis longicaudata (Serpentes: Xenodontinae) with comments on its hemipenial morphology and natural history. Herpetologica 67:318-331. http://dx.doi.org/10.1655/ HERPETOLOGICA-D-10-00043.1

DIXON, J.R. 1983. Systematics of Liophis reginae and L. williamsi (Serpentes, Colubridae), with a description of a new species. Ann. Carnegie Mus. 52(6):113-138.

DIXON, J.R. \& HENDRICKS, F. 1979. The wormsnakes (Family Typhlopidae) of the Neotropics, exclusive of the Antilles. Zool. Verh. 173:1-39.

DIXON, J.R., WIEST, J.A. \& CEI, J.M. 1993. Revision of the Neotropical snake genus Chironius Fitzinger (Serpentes, Colubridae). Monogr. / Mus. Reg. Sci. Nat. Torino XIII: 1-279.

FEITOSA, D.T., PRUDENTE, A.L.C. \& LIMA, A.C. 2007. Redescription and variation of Micrurus paraensis Cunha \& Nascimento 1973 (Serpentes: Elapidae). Zootaxa. 1470:35-45.

FERNANDES, D.S., GERMANO, V.J., FERNANDES, R. \& FRANCO, F.L. 2002. Taxonomic status and geographic distribution of the lowland species of the Liophis cobella group with comments on the species from the Venezuelan tepuis (Serpentes, Colubridae). Bol. Mus. Nac., N. S., Zool. 481:1-14.

FERNANDES, D.S., FRANCO, F.L. \& FERNANDES, R. 2004. Systematic revision of the genus Lachesis Daudin, 1803 (Serpentes, Viperidae). Herpetologica 60(2):245-260.

FERNANDES, D.S., MARQUES, O.A.V. \& ARGÔLO, A.J.S. 2010. A new species of Dipsas Laurenti from the Atlantic Forest of Brazil (Serpentes: Dipsadidae). Zootaxa 2691:57-66.

FRANÇA, F.G.R., MESQUITA, D.O. \& COLLI, G.R. 2006. A cheklist of snakes from Amazonian savannas in Brazil, housed in the coleção herpetológica da Universidade de Brasília, with new distribution records. Occ. Pap. Oklahoma Mus. Nat. Hist., Univ. 17:1-13.

FRANCO, F.L., FERNANDES, D.S. \& BENTIM, B.M. 2007. A new species of Hydrodynastes Fitzinger, 1843 from central Brazil (Serpentes: Colubridae: Xenodontinae). Zootaxa 1613:57-65.

FROTA, J.G. 2004. As serpentes da região de Itaituba, médio rio Tapajós, Pará, Brasil (Squamata). Comun. Mus. Ciênc. Tecnol. PUCRS, sér. Zool. 17(1):9-19.

FROTA, J.G. 2005. Nova espécie de Helicops Wagler, 1830 (Serpentes, Colubridae) do rio Tapajós, Amazônia, Brasil. Phyllomedusa 4(1):61-68.

FROTA, J.G., SANTOS-JUNIOR, A.P., CHALKIDIS, H.M. \& GUEDES, A.G. 2005. As serpentes da região do baixo rio Amazonas, oeste do Estado do Pará, Brasil (Squamata). Biociências 13(2):211-220.

GAINSBURY, A.M. \& COLLI, G.R. 2003. Lizard assemblages from natural cerrado enclaves in Southwestern Amazonia: the role of stochastic extinctions and isolation. Biotropica 35(4):503-519.

GRAZZIOTIN, F.G., ZAHER, H., MURPHY, R.W., SCROCCHI, G., BENAVIDES, M.A., ZHANG, Y. \& BONATTO, S.L. 2012. Molecular phylogeny of the New World Dipsadidae (Serpentes: Colubroidea): a reappraisal. Cladistics 1 2012:1-23. http://dx.doi.org/10.1111/j.10960031.2012.00393.x

GRIFFIN, L.E. 1916. A catalog of the Ophidia from South America at present (June, 1916) contained in the Carnegie museum, with descriptions of some new species. Mem. Carnegie Mus. 7(3):163-228.

GUSMÃO, A.C., CASAGRANDE, L.P., SILVA, A.M., CRISPIN, M.A., GARCIA, J.R.F., SOUZA, M.R., SUZECK, E. \& BERNARDE, P.S. 2011. Chironius exoletus (Vine Snake). Reproductive behavior. Herpetol. Rev. 42(2):284. 
HENDERSON, R.W. 1997. A taxonomic review of the Corallus hortulanus complex of Neotropical tree boas. Caribb. J. Sci. 33(3-4):198-221.

HENDERSON, R.W., PASSOS, P. \& FEITOSA, D. 2009. Geographic variation in the Emerald Treeboa, Corallus caninus (Squamata: Boidae). Copeia 2009:572-582. http://dx.doi.org/10.1643/CH-08-190

HOGE, A.R. \& NINA, A.C.M. 1964. Serpentes coletadas pelo Instituto Nacional de Pesquisas da Amazônia. Mem. Inst. Butantan. 30:71-96.

HOGE, A.R. \& ROMANO-HOGE, S.A.R.W.L. 1981. Poisonous snakes of the world. Part 1. Check list of the pit vipers, Viperoidea, Viperidae, Crotalinae. Mem. Inst. Butantan. 42-43:179-309.

HOGE, A.R., SANTOS, N.P., LOPES, L.A. \& SOUZA, I.M. 1973. Serpentes coletadas pelo projeto Rondon VII em Iauareté, Brasil. Mem. Inst. Butantan. 36:221-232.

JORGE-DA-SILVA JUNIOR, N. 1993. The snakes from Samuel hydroeletric power plant and vicinity, Rondônia, Brasil. Herpetol. Nat. Hist. 1:37-86.

JORGE-DA-SILVA JUNIOR, N., SILVA, H.L.R., RIBEIRO, R.S., SOUZA, I. \& SOUZA, C.A. 2005. Uma nova espécie do gênero Atractus Wagler, 1928 (Colubridae: Dipsadinae) do Cerrado do Brasil Central. Pap. Avul. Zool. 45(3):33-39.

KEISER JUNIOR, E.D. 1989. Oxybelis boulengeri Procter, a valid species of vine snake from South America. Copeia. 1989(3):764-768.

LEMA, T. 2004. Nova espécie de Apostolepis Cope do Estado de Rondônia, Brasil (Serpentes: Elapomorphinae). Comun. Mus. Ciênc. Tecnol. PUCRS, sér. Zool. 17(2):81-89.

LEMA, T. \& ALBUQUERQUE, N.R. 2010. The identity of Apostolepis pymi and placement of $A$. quinquelineata in the synonymy of $A$. nigrolineata (Serpentes, Xenodontinae). Biota neotrop. 10(1): http:// www.biotaneotropica.org.br/v10n1/pt/abstract?article+bn02810012010 (último acesso em 20/04/2011).

LEMA, T. \& RENNER, M.F. 1998. O status de Apostolepis quinquelineato Boulenger, 1896, A. pymi, Boulenger, 1903 e A. rondoni Amaral, 1925 (Serpentes, Colubridae, Elapomorphini). Biociências. 6(1):99-121.

LEMA, T., BERNARDE, P.S., MACEDO-BERNARDE, L.C., NASCIMENTO, P.F., TURCI, L.C.B. \& SANTOS, D.V. 2005. Ocorrência de Phalotris nasutus (Gomes, 1915) no estado de Rondônia, Brasil (Serpentes: Colubridae: Elapomorphinae). Comun. Mus. Ciênc. Tecnol. PUCRS, sér. Zool. 18(2):211-212.

LIMA, A.C. \& PRUDENTE, A.L.C. 2009. Morphological variation and systematics of Dipsas catesbyi (Sentzen, 1796) and Dipsas pavonina Schlegel, 1837 (Serpentes: Dipsadinae). Zootaxa 2203:31-48.

LYNCH, J.D. 2009. Snakes of the genus Oxyrhopus (Colubridae: Squamata) in Colombia: taxonomy and geographic variation. Pap. Avul. Zool. 49(25):319-337.

MACEDO-BERNARDE, L.C. 2006. Eunectes murinus (Linnaeus) (Serpentes, Boidae), preying activity. Pan-Am. J. aquat. Sci. 1(2):2.

MACEDO-BERNARDE, L.C. \& BERNARDE, P.S. 2005. Bothrops atrox (Common Lancehead) diet. Herpetol. Rev. 36(4):456.

MACEDO, L.C., BERNARDE, P.S. \& ABE, A.S. 2008. Lagartos (Squamata:Lacertilia) em áreas de floresta e de pastagem em Espigão do Oeste, Rondônia, sudoeste da Amazônia, Brasil. Biota Neotrop. 8(1): http://www.biotaneotropica.org.br/v8n1/pt/fullpaper?bn01108012008 (último acesso em 10/04/2011).

MACHADO, S.R. 1993. A new genus of Amazonian vine snake (Xenodontinae: Alsophiini). Acta Biol. Leopoldensia 15(2):99-108.

MARÇAL, A.S., GOMES, I.B.R. \& CORAGEM, J.T. (Orgs.). 2011. UHE Santo Antônio - Guia das espécies de fauna resgatadas. Scriba Comunicação Corporativa, Porto Velho, 327p.

MARTINS, M. \& OLIVEIRA, M.E. 1998. Natural history of snakes in forests of the Manaus region, Central Amazonia, Brazil. Herpetol. Nat. Hist. 6(2):78-150.

MOURA-LEITE, J.C. \& BERNARDE, P.S. 1999. Geographic distribution: Waglerophis merremii (Boipeva). Herpetol. Rev. 30:56.

NASCIMENTO, F.P., AVILA-PIRES, T.C.S. \& CUNHA, O.R. 1987. Os répteis da área de Carajás, Pará, Brasil (Squamata). II. Bol. Mus. Para. Emílio Goeldi, Sér. Zool. 3(1):33-65.
NASCIMENTO, F.P., ÁVILA-PIRES, T.C.S. \& CUNHA, O.R. 1988. Répteis Squamata de Rondônia e Mato Grosso coletados através do programa Polonoroeste. Bol. Mus. Para. Emílio Goeldi, Sér. Zool. 4:21-66.

NASCIMENTO, P.F., BERNARDE, P.S. \& BUZZETTI, D.R. C. 2008. Bothrops atrox (Amazonian Lancehead). Diet. Herpetol. Rev. 39(3):353.

NOGUEIRA, C., COLLI, G.R., COSTA, G.C. \& MACHADO, R.B. 2010. Diversidade de répteis Squamata e evolução do conhecimento faunístico no Cerrado. In Cerrado: conhecimento científico quantitativo como subsídio para ações de conservação (I.R. Diniz, J. Marinho-Filho, R.B. Machado, R.B. Cavalcanti, orgs.). Editora UnB, Brasília, p.333-375.

NOGUEIRA, C., RIBEIRO, S.C., GABRIEL C. \& COLLI, G.R. 2011. Vicariance and endemism in a Neotropical savanna hotspot: distribution patterns of Cerrado squamate reptiles. J. Biogeogr. 38(10):1907-1922. http://dx.doi.org/10.1111/j.1365-2699.2011.02538.x

OLIVEIRA, O.A. 2002. Geografia de Rondônia: Espaço e Produção. Dinâmica, Porto Velho, 160p.

O'SHEA, M.T. \& STIMSON, A.F. 1993. An aberrant specimen of Drymobius rhombifer (Colubridae: Colubrinae): a new generic record for Brazil. Herpetol. J. 3:70-71.

PASSOS, P. \& FERNANDES, D.S. 2005. Variation and taxonomic status of the aquatic coral snake Micrurus surinamensis (Cuvier, 1817) (Serpentes: Elapidae). Zootaxa 953:1-14.

PASSOS, P. \& FERNANDES, R. 2009. Revision of the Epicrates cenchria complex (Serpentes: Boidae). Herpetol. Monog. 22:1-30. http://dx.doi. org/10.1655/06-003.1

PASSOS, P., FERNANDES, D.S. \& CARAMASCHI, U. 2004. The taxonomic status of Leptognathus incertus Jan, 1863, with revalidation of Dipsas alternans (Fisher, 1885) (Serpentes: Colubridae: Dipsadinae). AmphibiaReptilia 25:381-393. http://dx.doi.org/10.1163/1568538042788951

PRUDENTE, A.L.C. \& PASSOS, P. 2008. A new species of Atractus Wagler, 1828 (Serpentes: Dipsadinae) from Guyana Plateau in Northern Brazil. J. Herpetol. 42:723-732. http://dx.doi.org/10.1670/07-115R3.1

PRUDENTE, A.L.C. \& PASSOS, P. 2010. A new cryptic species of Atractus from Brazilian Amazon. Copeia 2010(3):397-404. http://dx.doi. org/10.1643/CH-08-193

PRUDENTE, A.L.C., SANTOS-COSTA, M.C. 2005. Checklist of Snakes in the Eastern Amazon, Pará State, Brazil: Floresta Nacional de Caxiuanã Bol. Mus. Para. Emílio Goeldi, Sér. Zool. 3(1):243-251.

PRUDENTE, A.L.C., MOURA-LEITE, J.C. \& MORATO, S.A.A. 1998. Alimentação das espécies de Siphlophis Fitzinger (Serpentes: Colubridae: Xenodontinae: Pseudoboini). Revta Bras. Zool. 15:375-383. http://dx.doi. org/10.1590/S0101-81751998000200010

PRUDENTE, A.L.C., SILVA, M.A.A., ROCHA, W.A. \& FRANCO, F.L. 2008. Morphological variation in Xenoxybelis boulengeri (Procter, 1923) (Serpentes, Xenodontinae, Philodryadini). Zootaxa 1743:53-61.

PRUDENTE, A.L.C., MASCHIO, G.F., SANTOS-COSTA, M.C. \& FEITOSA, D.T. 2010. Serpentes da Bacia Petrolífera de Urucu, Município de Coari, Amazonas, Brasil. Acta Amazôn. 40(2):381-386.

RODRIGUES, M.T. 2005. Conservação dos répteis brasileiros: os desafios para um país megadiverso. Megadiversidade. 1(1):87-94.

ROZE, J.A. 1994. Notes on taxonomy of venomous coral snakes (Elapidae) of South America. Bull. Maryland Herp. Soc. 30(4):177-185.

ROZE, J.A. 1996. Coral snakes of the Americas: Biology, identification and venoms. Krieger Publishing Company, Malabar, 328p.

ROZE, J.A. \& JORGE-DA-SILVA JUNIOR, N. 1990. Coral snakes (Serpentes, Elapidae) from hydroelectric power plant of Samuel, Rondônia, Brazil, with a description of a new species. Bull Maryland Herp. Soc. 26(4):168-175.

SANTOS-JUNIOR, A.P., DI-BERNARDO, M. \& LEMA, T. 2008. New species of the Taeniophalus occipitalis Group (Serpentes, Colubridae) from Eastern Amazonia, Brazil. J. Herpetol. 42(3):419-426.

SCARTOZZONI, R.R., TREVINE, V.C. \& GERMANO, V.J. 2010. Reptilia, Squamata, Serpentes, Dipsadidae, Pseudoeryx plicatilis (Linnaeus, 1758): new records and geographic distribution map. CheckList. 6(4):534-537.

SILVA, M.V., SOUZA, M.B. \& BERNARDE, P.S. 2010. Riqueza e dieta de serpentes do Estado do Acre, Brasil. Rev. Bras. Zoocienc. 12(2):55-66. 
Bernarde, P.S. et al.

TURCI, L.C.B. \& BERNARDE, P.S. 2008. Levantamento herpetofaunístico em uma localidade no município de Cacoal, Rondônia, Brasil. Bioikos. 22:101-108.

TURCI, L.C.B. \& BERNARDE, P.S. 2009. Vertebrados Atropelados na Rodovia Estadual 383 em Rondônia, Brasil. Biotemas. 22(1):121-127.

VANZOLINI, P.E. 1986. Levantamento herpetológico da área do Estado de Rondônia sob a influência da rodovia Br-364. Ministério da Ciência e Tecnologia, Brasília, 50p. (Relatório de pesquisa, Programa Polonoroeste/ Ecologia Animal, n. 1).

VOGT, R.C., FERRARA, C.R., BERNHARD, R., CARVALHO, V., BALENSIEFER, D.C., BONORA, L. \& NOVELLE, S.M.H. 2007. Herpetofauna. In Biodiversidade do Médio Madeira: Bases Científicas para Propostas de Conservação (L.R. Py-Daniel, C.P. Deus, A.L. Henriques, D.M. Pimpão \& O.M. Ribeiro, orgs.). MMA, MCT, Manaus v. 29, p. 127-143.

XAVIER-DA-SILVA, V. \& RODRIGUES, M.T. 2008. Taxonomic revision of the Bothrops neuwiedi complex (Serpentes, Viperidae) with description of a new species. Phyllomedusa. 7(1):45-90.
WHITTAKER, A. 1996. Observations of a group of red necked aracaris (Pteroglossus bitorquatus) attacking a vine snake (Oxybelis fulgidus) in Rondônia, Brazil. Ornitol. Neotrop. 7:67-68.

ZAHER, H. 1996. A new genus and species of Pseudoboine snake, with a revision of the genus Clelia (Serpentes, Xenodontinae). Boll. / Mus. Reg. Sci. Nat. 14(2):289-337.

ZAHER, H., SOUZA, I., GOWER, D.J., HINGST-ZAHER, E. \& JORGEDA-SILVA JUNIOR, N. 2005. Redescription of Atractus albuquerquei (Serpentes, Colubridae, Dipsadinae), with comments on geographical distribution and intraspecific variation. Pap. Avul. Zool. 45:19-32.

ZAHER, H., OLIVEIRA, M.E. \& FRANCO, F.L. 2008. A new, brightly colored species of Pseudoboa Schneider, 1801 from the Amazon Basin (Serpentes, Xenodontinae). Zootaxa 1674:27-37.

ZIMMERMANN, B.L. \& RODRIGUES, M.T. 1990. Frogs, snakes, and lizards of the INPA/WWF reserves near Manaus, Brazil: Four Neotropical Rainforests (In A.H. Gentry ed.).Yale University Press, New Haven, p.426-454.

YUKI, R.N., GALATTI, U. \& ROCHA, R.A.T. 1999. Contribuição ao conhecimento da fauna de Squamata de Rondônia, Brasil, com dois novos registros. Bol. Mus. Para. Emílio Goeldi, Sér. Zool. 15(2):181-193.

Recebido em 20/05/2011

Versão reformulada recebida em 07/07/2012

Publicado em 09/07/2012 